\title{
Design of a Dynamical Core Based on the Nonhydrostatic "Unified System" of Equations*
}

\author{
Celal S. KonOR \\ Department of Atmospheric Science, Colorado State University, Fort Collins, Colorado
}

(Manuscript received 4 June 2013, in final form 25 July 2013)

\begin{abstract}
This paper presents the design of a dry dynamical core based on the nonhydrostatic "unified system" of equations. The unified system filters vertically propagating acoustic waves. The dynamical core predicts the potential temperature and horizontal momentum. It uses the predicted potential temperature to determine the quasi-hydrostatic components of the Exner pressure and density. The continuity equation is diagnostic (and used to determine the vertical mass flux) because the time derivative of the quasi-hydrostatic density is obtained from the predicted potential temperature. The nonhydrostatic component of the Exner pressure is obtained from an elliptic equation. The main focus of this paper is on the integration procedure used with this unique dynamical core. In the implementation described in this paper, height is used as the vertical coordinate, and the equations are vertically discretized on a Lorenz-type grid. Cartesian horizontal coordinates are used along with an Arakawa $\mathrm{C}$ grid. A detailed description of the discrete equations is presented in the supplementary material, along with the rationale behind the decisions made during the discretization process. Only a short description is given here. To demonstrate that the model is capable of simulating a wide range of dynamical scales, the results from cyclone- and cloud-scale simulations are presented. The solutions obtained for the selected cloud-scale simulations are compared to those from a fully compressible, anelastic, and pseudo-incompressible models that (as far as possible) uses the same schemes used in the unified dynamical core. The results show that the unified dynamical core performs reasonably well in all these experiments.
\end{abstract}

\section{Introduction}

Almost all of the nonhydrostatic models developed so far use the fully compressible, anelastic, or pseudoincompressible systems of equations in their dynamical cores. Examples include the Nonhydrostatic Icosahedral Atmospheric Model (NICAM; Satoh et al. 2008), the Model for Prediction Across Scales (MPAS; Skamarock et al. 2012), the Weather Research and Forecasting Model (WRF; Skamarock et al. 2005), the Met Office Unified Model (UKMO-UM; Staniforth et al. 2003; Davies et al. 2005), the Icosahedral Nonhydrostatic general circulation

\footnotetext{
* Supplemental information related to this paper is available at the Journals Online website: http://dx.doi.org/10.1175/MWR-D13-00187.s1.

Corresponding author address: Dr. Celal S. Konor, Department of Atmospheric Science, Colorado State University, Fort Collins, CO 80523-1371.

E-mail: csk@atmos.colostate.edu
}

model of the Max Planck Institute/Deutscher Wetterdienst (ICON-MPI/DWD; Bonaventura 2004), the National Centers for Environmental Prediction (NCEP) Nonhydrostatic Mesoscale Model (NMM; Janjic et al. 2001; Janjic 2003), the Canadian Meteorological CenterMeteorological Research Branch (CMC-MRB) Global Environmental Multiscale Model (GEM; Yeh et al. 2002), the Eulerian/semi-Lagrangian fluid solver (EULAG; Abiodun et al. 2008; Smolarkiewicz et al. 2001; Smolarkiewicz 2010), and others. The fully compressible system includes rapidly propagating acoustic waves of all scales. Without a special treatment, the acoustic waves can restrict the time step to seconds, depending on the spatial resolution. The use of implicit differencing in the vertical is often used to allow longer time steps with vertically propagating acoustic waves. A split-explicit or semi-implicit method is used in the treatment of the horizontally propagating acoustic waves [for the splitexplicit method, see Klemp and Wilhelmson (1978); Skamarock and Klemp (2008), and, for the semi-implicit method, see Tapp and White (1976); Kwizak and Robert 
(1971); Robert (1982); Côté et al. (1998); Cullen (1990)]. An extensive review of these methods and other numerical methods used in nonhydrostatic models can be found in Steppeler et al. (2003). The anelastic system, on the other hand, excludes the acoustic waves all together by neglecting the time change of density in the continuity equation (Ogura and Phillips 1962; Lipps and Hemler 1982; Bannon 1996). Then, a reference state density that changes only with height replaces the density. By neglecting the time change of density, the elasticity due to the thermal expansion is excluded, which introduces errors. [The term "error" means the deviation of solution from the fully compressible system as inferred from a normal mode analysis. See Davies et al. (2003); Arakawa and Konor (2009); Klein et al. (2010); Dukowicz (2013).] Some of these errors are avoided in the pseudo-incompressible system (Durran 1989, 2008), but others such as the difficulties in accurately simulating compressible large-scale Rossby waves remain (Arakawa and Konor 2009).

Arakawa and Konor (2009) introduced a new nonhydrostatic system of equations that unifies the anelastic and quasi-hydrostatic systems. The intended application of this "unified system of equations" is global cloud resolving models. In certain aspects, the unified system resembles the system introduced by Laprise (1992) and the one proposed by Miller (1974), in which the fully compressible and quasi-hydrostatic systems of equations are unified to include nonhydrostatic effects into the quasi-hydrostatic models with pressure based vertical coordinate. Marshall et al. (1997) introduced a system for ocean models that unifies the anelastic and quasihydrostatic systems for the same purpose. The unified system we are presenting here filters the vertically propagating acoustic waves while maintaining elasticity due to thermal expansion, by adopting the continuity equation of the quasi-hydrostatic system. The continuity equation is diagnostic (used to determine the vertical mass flux) because the quasi-hydrostatic density is obtained from the predicted potential temperature (and surface Exner pressure). No reference state is needed and no approximations are made in either the thermodynamic or the (horizontal and vertical) momentum equations. Total energy conservation is achieved by properly determining the mean value of the nonhydrostatic Exner pressure that is obtained by solving a threedimensional elliptic equation. The mean value of the nonhydrostatic Exner pressure is not used in the dynamical core presented here because it does not contribute to the dynamics. It would be needed, however, in a parameterization of cloud processes. Arakawa and Konor (2009) show, through a normal mode analysis, that the unified system is free of errors seen with the anelastic and pseudo-incompressible systems.
As a consequence of the filtering, the unified system, like the anelastic and pseudo-incompressible systems, requires the solution of an elliptic equation. In the largescale applications, in which the quasi-hydrostatic dynamics dominates over the nonhydrostatic dynamics, the elliptic equation of the unified system, however, needs to do little or no work because it is used to diagnose only the nonhydrostatic contribution to the (Exner) pressure. In effect, the unified system automatically includes a preconditioning process for the elliptic equation, which speeds up the convergence of the solution [P. K. Smolarkiewicz 2010, personal communication; see e.g., Skamarock et al. (1997)]. The generalized pseudo-incompressible system by Durran (2008) eases the workload on the elliptic solver because it allows the use of a three-dimensional timedependent reference state.

The purpose of this paper is to present the practical design of a dynamical core based on the unified system, using the height coordinate. The main focus is on the integration procedure, which is unique because, as stated earlier, the continuity equation is diagnostic, even though the time change of density is included. The vertical mass flux is diagnostically obtained from the continuity equation, while the time change of the quasi-hydrostatic density is determined from the predicted potential temperature. Another feature of this integration procedure is that the (Exner) pressure, density, and temperature are split into their quasi-hydrostatic and nonhydrostatic components, which are determined through different procedures.

The finite-difference schemes used in the dynamical core are conventional; they are not particularly new or sophisticated, and all of them were originally designed for use in quasi-hydrostatic models. We follow this approach in part because we want to demonstrate that a quasi-hydrostatic model can be easily converted to a nonhydrostatic unified model through add-on modules. The schemes used here can produce good solutions, as will be evident from the test simulations presented.

The unified dynamical core uses a Lorenz grid (Lorenz 1960) in the vertical discretization and an Arakawa C grid (Arakawa and Lamb 1977; Arakawa 1988) in the horizontal discretization. The Lorenz grid has been chosen for this version because it is the most commonly used vertical grid. Future versions of the dynamical core may use a Charney-Phillips-type grid to take advantage of its nice properties (Arakawa and Konor 1996). The horizontal domain of the model can be a square or rectangle on an irrotational plane, $f$ or $\beta$ plane. It can use periodic lateral boundaries in $x$ and $y$ directions (for irrotational case) or a combination of flux-free north and south boundaries and cyclic zonal boundaries (for $f$ or $\beta$ plane). 
The dynamical core presented here has been designed for tests to demonstrate that the simulations are comparable to those obtained by the fully compressible dynamical cores in small domains. Two-dimensional cloud-scale simulations of warm and cold bubbles are directly compared to those obtained with a fully compressible dynamical core constructed by using (as far as possible) same schemes. Selected warm bubble simulations are also compared to the ones obtained with the anelastic and pseudo-incompressible dynamical cores. The three-dimensional $\beta$-plane simulations can be compared to the simulations performed by Ullrich and Jablonowski (2012). We do not intent to use the unified system in small-domain applications. For that purpose the fully compressible, anelastic, and pseudo-incompressible systems are already available, and work well. Our goal is to use the unified system in a global model, in which the unified system automatically switches between the nonhydrostatic and quasi-hydrostatic systems depending on the resolution used. Based on the work reported in the present paper, a global dynamical core based on the unified system has already been constructed and is currently being tested. The results will be presented elsewhere.

This paper is organized as follows. Section 2 presents the continuous equations of the unified system. The discretization of the equations is briefly discussed in section 3 . A detailed discussion of the discrete equations, including their derivation, is presented in the supplementary material. For clarity, the discrete equations are presented in the same order in which they are used in the model. Section 4 shows results from the extratropical cyclogenesis on a midlatitude $\beta$ plane and cloud-scale warm and cold bubble simulations without rotation. For comparison purposes, section 4 also shows the same fields simulated by fully compressible, anelastic, and pseudo-incompressible dynamical cores constructed by using the schemes used in the unified dynamical core for selected cases.

\section{The unified system of equations}

The unified system of equations is described in detail by Arakawa and Konor (2009). In this section, the basic continuous equations of the system in a height vertical coordinate are reintroduced and their important features are briefly discussed for convenience. For simplicity, the virtual temperature effect is neglected throughout this paper. The inclusion of virtual temperature effect is discussed in the supplementary material.

The thermodynamic quantities used in the unified system, with the exception of the potential temperature $(\theta)$ and height $(z)$, are formally defined by

$$
\begin{aligned}
& \rho \equiv \rho_{\mathrm{qs}}+\delta \rho, \quad \pi \equiv \pi_{\mathrm{qs}}+\delta \pi, \quad p \equiv p_{\mathrm{qs}}+\delta p, \quad \text { and } \\
& T \equiv T_{\mathrm{qs}}+\delta T,
\end{aligned}
$$

where $\rho$ is the density, $\pi$ is the Exner pressure given by $\pi \equiv\left(p / p_{00}\right)^{\kappa}, p$ is the pressure, $p_{00}$ is a standard pressure ( $=1000 \mathrm{hPa}), \kappa \equiv R / c_{p}, R$ is the gas constant for the dry air, $c_{p}$ is the specific heat of dry air at constant pressure, and $T$ is the temperature. In these equations, the subscript qs and prefix $\delta$ denote the quasi-hydrostatic and nonhydrostatic portions of each quantity, respectively. In Eq. (2.1), the quasi-hydrostatic temperature is defined by $T_{\mathrm{qs}} \equiv \pi_{\mathrm{qs}} \theta$. It is important to recognize that the quasi-hydrostatic values in Eq. (2.1) do not represent a fixed basic or reference state because these quantities are all obtained from the three-dimensionally predicted potential temperature. This point will be further discussed in section 2e. It should also be noted that the quasi-hydrostatic quantities in the unified system are not necessarily identical to those in the quasi-hydrostatic system because the potential temperature and height have nonhydrostatic components in them. In the unified system, the quasi-hydrostatic fields correspond to a threedimensional time-dependent balanced state in some sense, on which the nonhydrostatic deviations are added. In this sense, the unified system resembles to those introduced by Marshall et al. (1997), Smolarkiewicz et al. (2001), and Durran (2008). The balanced state becomes identical to the true quasi-hydrostatic state for the largescale applications in the unified system.

\section{a. The equation of state}

Two equations of state are used in the unified system. The first is

$$
p=\rho R T,
$$

which is free from any approximation. The other is the "quasi-hydrostatic state equation" given by

$$
p_{\mathrm{qs}}=\rho_{\mathrm{qs}} R T_{\mathrm{qs}},
$$

which relates the quasi-hydrostatic pressure, density, and temperature to each other.

\section{b. The continuity equation}

The continuity equation of the unified system is given by

$$
\nabla_{H} \cdot\left(\rho_{\mathrm{qs}} \mathbf{v}\right)+\frac{\partial}{\partial z}\left(\rho_{\mathrm{qs}} w\right)=-\frac{\partial \rho_{\mathrm{qs}}}{\partial t},
$$

where $t$ is time, $\nabla_{H}$ is the horizontal del operator, $\mathbf{v}$ is the horizontal velocity, $z$ is the height and the vertical 
coordinate, and $w$ is the vertical velocity. At the upper and lower boundaries $\left(z=z_{T}\right)$ and $\left(z=z_{S}\right)$, respectively, we define

$$
w_{S}=w_{T}=0
$$

because the surface topography is not considered in this study. With the inclusion of surface topography, $z_{S}$ becomes a horizontally varying constant and the vertical velocity at the surface is defined by $w_{S} \equiv \mathbf{v}_{S} \cdot \nabla_{H} z_{S}$. In the unified system, the continuity equation in Eq. (2.3) is not used to predict $\rho_{\mathrm{qs}}$, but instead is used to diagnostically determine the vertical mass flux. The time derivative of $\rho_{\mathrm{qs}}$ is obtained from the predictions of the potential temperature and the surface quasi-hydrostatic Exner pressure, as discussed in section 2e.

The continuity equation in Eq. (2.3) introduces the only approximation that distinguishes the unified system from the fully compressible system. The unified continuity equation in Eq. (2.3) is obtained by assuming $\delta \rho / \rho_{\mathrm{qs}} \ll 1$ and dropping $\partial \delta \rho / \partial t$ to filter the vertically propagating acoustic waves. However, by keeping the term $\partial \rho_{\mathrm{qs}} / \partial t$, the effect of elasticity due to thermal expansion is still included in the system. No approximations are made in the thermodynamic equation or the horizontal and vertical momentum equations, which will be discussed next. Arakawa and Konor (2009) show, through a normal mode analysis, that the approximations made in the continuity equation are sufficient to filter the vertically propagating acoustic waves without affecting the other modes.

\section{c. The thermodynamic equation}

The thermodynamic equation is given by

$$
\frac{D \theta}{D t}=\frac{Q}{c_{p} \pi}
$$

where $D / D t \equiv \partial / \partial t+\mathbf{v} \cdot \nabla_{H}+w \partial / \partial z$ is the material time derivative. In Eq. (2.5), $Q$ is the heating rate per unit mass.

\section{d. The horizontal and vertical momentum equations}

The vector-invariant form of the horizontal momentum equation is given by

$$
\begin{aligned}
\frac{\partial \mathbf{v}}{\partial t}= & -q \mathbf{k} \times\left(\rho_{\mathrm{qs}} \mathbf{v}\right)-w \frac{\partial \mathbf{v}}{\partial z}-\nabla_{H} K \\
& -c_{p} \theta \mathbf{\nabla}_{H}\left(\pi_{\mathrm{qs}}+\delta \pi\right)+\mathbf{F}_{H},
\end{aligned}
$$

where $q$ is the potential vorticity defined by $q \equiv(\zeta+f) / \rho_{\mathrm{qs}}$, $\zeta$ is the vorticity defined by $\zeta \equiv \mathbf{k} \cdot \nabla_{H} \times \mathbf{v}, f$ is the Coriolis parameter and $\mathbf{k}$ is the unit vector in the vertical direction,
$K$ is kinetic energy defined by $K \equiv(1 / 2) \mathbf{v}^{2}$, and $\mathbf{F}_{H}$ represents the physical processes, such as the horizontal friction and diffusion of $\mathbf{v}$.

We formally write the vertical momentum equation as

$$
\frac{D w}{D t}+c_{p} \theta \frac{\partial \delta \pi}{\partial z}=F_{z},
$$

where $F_{z}$ represents the physical processes, such as the vertical friction and diffusion of $w$. To maintain consistency with the boundary conditions given by Eq. (2.4), the vertical momentum equations at the upper and lower boundaries are modified to

$$
\left(c_{p} \theta \frac{\partial \delta \pi}{\partial z}\right)_{S}=\left(F_{z}\right)_{S} \quad \text { and } \quad\left(c_{p} \theta \frac{\partial \delta \pi}{\partial z}\right)_{T}=\left(F_{z}\right)_{T},
$$

respectively. Note that $\left(F_{z}\right)_{S}=\left(F_{z}\right)_{T}=0$ can be assumed. Since the vertical velocity in the unified system is diagnostically determined through the continuity equation given by Eq. (2.3), the vertical momentum equation in Eq. (2.7) is not actually used to predict the vertical momentum. Equation (2.8) is needed for the derivation of the elliptic equation that determines nonhydrostatic Exner pressure $\delta \pi$, which is discussed in section $2 \mathrm{f}$.

\section{e. Determination of the quasi-hydrostatic Exner pressure and the quasi-hydrostatic density}

The "hydrostatic equation" can be written in two forms:

$$
\partial p_{\mathrm{qs}} / \partial z=-g \rho_{\mathrm{qs}}
$$

and

$$
\partial \pi_{\mathrm{qs}} / \partial z=-g / c_{p} \theta .
$$

We refer to these equations as the hydrostatic equations for convenience. It should be noted that they are different than those used in the quasi-hydrostatic system because of the reasons stated at the beginning of section 2. In the unified system, they are used to diagnose the quasi-hydrostatic (Exner) pressure. Although Eqs. (2.9a) and (2.9b) are not independent equations, we need both of them to determine $\partial\left(\pi_{\mathrm{qS}}\right)_{S} / \partial t$ and $\partial\left(\pi_{\mathrm{qs}}\right)_{T} / \partial t$ because $\partial\left(\pi_{\mathrm{qs}}\right)_{T} / \partial t$ is not a correct condition for the height coordinate (p. 714 of Arakawa and Konor 2009). By first taking the derivative of Eqs. (2.9a) and (2.9b) with respect to time and then integrating these equations with respect to height starting at the surface $(z=$ $z_{S}$ ), the prediction equations for the quasi-hydrostatic 
pressure and the quasi-hydrostatic Exner pressure can be written as

$$
\frac{\partial p_{\mathrm{qs}}}{\partial t}-\frac{\partial\left(p_{\mathrm{qs}}\right)_{S}}{\partial t}=-g \int_{z=z_{s}}^{z} \frac{\partial \rho_{\mathrm{qs}}}{\partial t} d z
$$

and

$$
\frac{\partial \pi_{\mathrm{qs}}}{\partial t}-\frac{\partial\left(\pi_{\mathrm{qs}}\right)_{S}}{\partial t}=\frac{g}{c_{p}} \int_{z=z_{S}}^{z} \frac{1}{\theta^{2}} \frac{\partial \theta}{\partial t} d z,
$$

respectively. By using the unified continuity equation in Eq. (2.3) with the boundary conditions given by Eq. (2.4) and $\partial p_{\mathrm{qs}} / \partial t=\left(p_{\mathrm{qs}} / \kappa \pi_{\mathrm{qs}}\right) \partial \pi_{\mathrm{qs}} / \partial t$ in Eq. (2.10a) and extending the integrations to the upper boundary $\left(z=z_{T}\right)$ in both Eqs. (2.10a) and (2.10b), the integrals can be written as

$$
\left(p_{\mathrm{qs}} / \kappa \pi_{\mathrm{qs}}\right)_{T} \frac{\partial\left(\pi_{\mathrm{qs}}\right)_{T}}{\partial t}-\left(p_{\mathrm{qs}} / \kappa \pi_{\mathrm{qs}}\right)_{S} \frac{\partial\left(\pi_{\mathrm{qs}}\right)_{S}}{\partial t}=-g \frac{\partial M}{\partial t},
$$

where

$$
\partial M / \partial t=-\nabla_{H} \cdot \int_{z=z_{S}}^{z_{T}}\left(\rho_{\mathrm{qs}} \mathbf{v}\right) d z
$$

and

$$
\frac{\partial\left(\pi_{\mathrm{qs}}\right)_{T}}{\partial t}-\frac{\partial\left(\pi_{\mathrm{qs}}\right)_{S}}{\partial t}=\frac{g}{c_{p}} \int_{z=z_{S}}^{z_{T}} \frac{1}{\theta^{2}} \frac{\partial \theta}{\partial t} d z
$$

respectively. In Eq. (2.11b), $M \equiv \int_{z=z S}^{z_{T}} \rho_{\mathrm{qS}} d z$ is the column mass. By eliminating $\partial\left(\pi_{\mathrm{qs}}\right)_{T} / \partial t$ between Eqs. (2.11a) and (2.11c), the prediction equation for the surface quasihydrostatic Exner pressure is obtained as

$\frac{\partial\left(\pi_{\mathrm{qs}}\right)_{S}}{\partial t}=\frac{\left(p_{\mathrm{qs}} / \kappa \pi_{\mathrm{qs}}\right)_{T} \frac{g}{c_{p}} \int_{z=z_{S}}^{z_{T}}\left(1 / \theta^{2}\right)(\partial \theta / \partial t) d z+g \partial M / \partial t}{\left(p_{\mathrm{qs}} / \kappa \pi_{\mathrm{qs}}\right)_{S}-\left(p_{\mathrm{qs}} / \kappa \pi_{\mathrm{qs}}\right)_{T}}$.

By using Eq. (2.12) in Eq. (2.10b), the prediction equation for the quasi-hydrostatic Exner pressure at the interior points can be obtained.

Next the equation that determines the quasi-hydrostatic density is derived. By applying $\pi_{\mathrm{qs}} \equiv\left(p_{\mathrm{qs}} / p_{00}\right)^{\kappa}$ and $T_{\mathrm{qs}} \equiv \pi_{\mathrm{qs}} \theta$ to $p_{\mathrm{qs}}=\rho_{\mathrm{qs}} R T_{\mathrm{qs}}$, the "quasi-hydrostatic" state equation of the unified system can be written as

$$
\rho_{\mathrm{qs}}=\frac{p_{00} \pi_{\mathrm{qs}}^{(1-\kappa) / \kappa}}{R \theta} .
$$

By taking the derivative of Eq. (2.13) with respect to time, the prediction equation for the quasi-hydrostatic density is obtained as

$$
\frac{\partial \rho_{\mathrm{qs}}}{\partial t}=\rho_{\mathrm{qs}}\left(\frac{1-\kappa}{\kappa} \frac{1}{\pi_{\mathrm{qs}}} \frac{\partial \pi_{\mathrm{qs}}}{\partial t}-\frac{1}{\theta} \frac{\partial \theta}{\partial t}\right) .
$$

By determining $\partial \rho_{\mathrm{qs}} / \partial t$ through Eq. (2.14) instead of the continuity equation in Eq. (2.3), the elasticity due to the thermal expansion is maintained in the system, while elasticity due to dynamic compressibility represented by $\partial \delta \rho / \partial t$ is removed.

A summary of the prediction procedure of the quasihydrostatic variables is as follows: at the beginning $(t=0)$, the main prognostic variables $\theta, \mathbf{v}, w$, and $\left(\pi_{\mathrm{qs}}\right)_{S}$ are given and, $\pi_{\mathrm{qs}}$ and $\rho_{\mathrm{qs}}$ are obtained from these main prognostic variables using Eqs. (2.9b) and (2.13), respectively. First, $\partial \theta / \partial t$ is determined from Eq. (2.5) and, at the same time, $\nabla_{H} \cdot \int_{z=z S}^{z_{T}}\left(\rho_{\mathrm{qs}} \mathbf{v}\right) d z$ is calculated and $\partial M / \partial t$ is determined. These two quantities are used in Eq. (2.12) to obtain $\partial\left(\pi_{\mathrm{qs}}\right)_{S} / \partial t$, and then $\partial \pi_{\mathrm{qs}} / \partial t$ is obtained from Eq. (2.10b). Using $\partial \theta / \partial t$ and $\partial \pi_{\mathrm{qs}} / \partial t$ in Eq. (2.14), the time change of quasi-hydrostatic density $\partial \rho_{\mathrm{qs}} / \partial t$ is determined. Note that if $w$ is not available at the beginning, it can be determined by using $\mathbf{v}$ in the continuity equation in Eq. (2.3) by assuming $\partial \rho_{\mathrm{qs}} / \partial t=0$.

\section{f. Determination of the nonhydrostatic Exner pressure and other nonhydrostatic quantities}

The multistep procedure used to derive the elliptic equation that determines $\delta \pi$ is as follows. (i) Through $\nabla_{H} \cdot\left[\rho_{\mathrm{qs}} \times\right.$ Eq. (2.6)], an equation that predicts horizontal mass flux convergence is obtained. (ii) Through $\partial\left[\rho_{\mathrm{qs}} \times\right.$ Eq. (2.7) $] / \partial z$, an equation that predicts vertical mass flux convergence is obtained. (iii) The time derivative of the unified continuity equation in Eq. (2.3) is taken. (iv) Finally, $\partial\left[\nabla_{H} \cdot\left(\rho_{\mathrm{qs}} \mathbf{v}\right)\right] / \partial t$ and $\partial\left[\partial\left(\rho_{\mathrm{qs}} w\right) / \partial z\right] / \partial t$ are eliminated between the equations obtained by (i), (ii), and by (iii). After arranging the terms, the threedimensional elliptic equation can be written as

$$
\begin{aligned}
\nabla_{H} & \cdot\left(\rho_{\mathrm{qs}} c_{p} \theta \nabla_{H} \delta \pi\right)+\frac{\partial}{\partial z}\left(\rho_{\mathrm{qs}} c_{p} \theta \frac{\partial \delta \pi}{\partial z}\right) \\
= & -\nabla_{H} \cdot\left(\rho_{\mathrm{qs}} c_{p} \theta \nabla_{H} \pi_{\mathrm{qs}}\right)-\nabla_{H} \cdot\left(\rho_{\mathrm{qs}} \mathbf{G}_{H}\right) \\
& -\frac{\partial\left(\rho_{\mathrm{qs}} G_{z}\right)}{\partial z}+\frac{\partial^{2} \rho_{\mathrm{qs}}}{\partial t^{2}}
\end{aligned}
$$

where

$$
\mathbf{G}_{H} \equiv q \mathbf{k} \times\left(\rho_{\mathrm{qs}} \mathbf{v}\right)+w \frac{\partial \mathbf{v}}{\partial z}+\nabla_{H} K-\mathbf{F}_{H}-\frac{\mathbf{v}}{\rho_{\mathrm{qs}}} \frac{\partial \rho_{\mathrm{qs}}}{\partial t}
$$

and 
(a) Vertical L-Grid

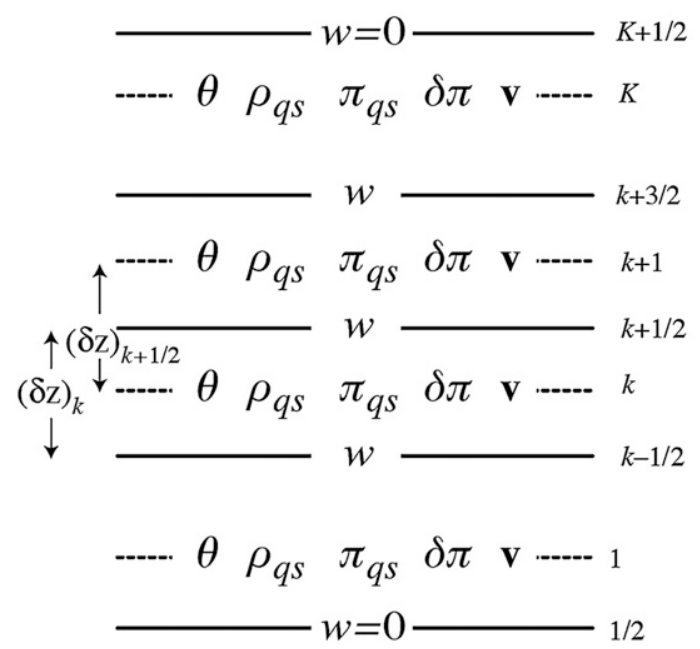

(b) Horizontal C-Grid

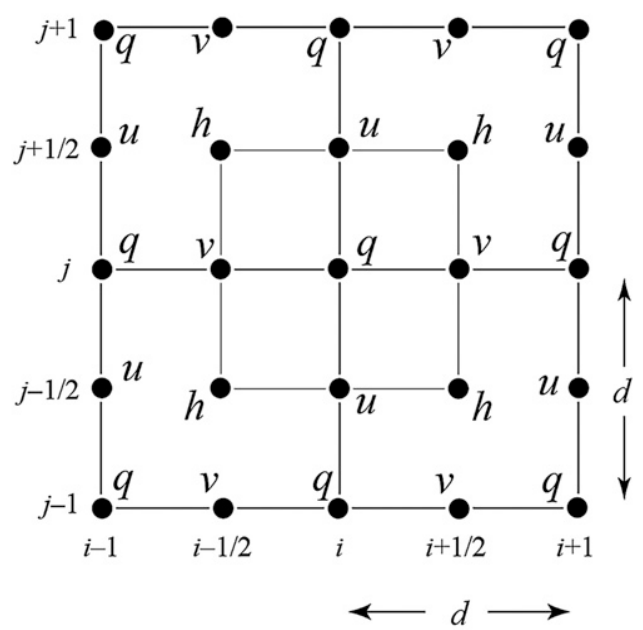

FIG. 1. (a) Vertical Lorenz grid and (b) horizontal $\mathrm{C}$ grid used in the discretization of equations. On the horizontal grid, $h$ points contain variables including $\theta, \pi_{\mathrm{qs}}, \delta \pi, \rho_{\mathrm{qs}}, T$, and $w ; q$ points contain the potential vorticity, vorticity, and Coriolis parameter.

$G_{z} \equiv \frac{1}{\rho_{\mathrm{qs}}} \nabla_{H} \cdot\left(w \rho_{\mathrm{qs}} \mathbf{v}\right)+\frac{1}{\rho_{\mathrm{qs}}} \frac{\partial}{\partial z}\left(\rho_{\mathrm{qs}} w w\right)-F_{Z}$

The upper and lower boundary conditions are given by Eq. (2.8).

\section{Discretization of the unified system of equations}

This section discusses the discretization of the equations presented in section 2 . The vertical and horizontal grids are introduced in section $3 \mathrm{a}$. The discretized equations are presented following the order in which they are used in the model. For clarity, a summary of the integration procedure is presented at the end of this section.

The dynamical core uses third-order (or higher order) schemes for the discretization of various horizontal and vertical fluxes, depending on applicability. The horizontal momentum equation is discretized using the fourth-order potential enstrophy-conserving scheme of Takano and Wurtele (1982), modified to dissipate potential enstrophy. For the time discretization of the advection of potential temperature and momentum, a second-order Adams-Bashforth scheme is selected, which has been successfully used in dynamical cores because it yields effectively stable solutions, is easy to implement, and is computationally efficient compared to similar schemes. For the time discretization of the pressure gradient force, we use the economical implicit time integration scheme of Mesinger and Arakawa (1976), which can yield stable (neutral) solutions for the Courant numbers up to one for the Lamb wave. Note that the
Lamb wave is the fastest mode in the unified system. A detailed description of the discretization is discussed in the supplementary material. Only a short description will be given here.

\section{a. Vertical and horizontal grids}

The first decision we make in the discretization of the equations is the choice of the vertical and horizontal grids. For the vertical grid, the Lorenz grid ( $\mathrm{L}$ grid) is selected although the Charney-Phillips grid (CP grid) has very important advantages over the $\mathrm{L}$ grid [see Arakawa and Konor (1996) for a comparison of the performances of the two grids]. The unified system does not favor one over the other. The main reason to choose the $\mathrm{L}$ grid for the first dynamical core constructed with the unified model is that the most atmosphere modelers are more familiar with the $\mathrm{L}$ grid. In the future, a version of the dynamical core using the $\mathrm{CP}$ grid is planned. On the $\mathrm{L}$ grid, the potential temperature, quasi-hydrostatic Exner pressure, quasi-hydrostatic density, nonhydrostatic Exner pressure, and horizontal velocities are predicted at the model layers, which are indexed with integers (dashed lines in Fig. 1a). The vertical velocity is diagnosed at the interfaces of the model layers, which are indexed with half integers (solid lines in Fig. 1a). The uppermost and lowermost interfaces coincide with the bottom and top boundaries, respectively. The vertical thicknesses of the layers are defined as the distance between the interfaces above and below, that is, $(\delta z)_{k} \equiv z_{k+1 / 2}-z_{k-1 / 2}$, where $z_{k+1 / 2}$ are the heights of the interfaces from surface to the top. The vertical grid spacings for the interfaces are 
determined from $(\delta z)_{k+1 / 2} \equiv z_{k+1}-z_{k}$ for the interior interfaces, where $z_{k}$ are the heights of the layers. At the bottom and top boundaries, $(\delta z)_{1 / 2} \equiv z_{1}-z_{1 / 2}$ and $(\delta z)_{K+1 / 2} \equiv$ $z_{K+1 / 2}-z_{K}$ are used, respectively. The superscript $S$ and $1 / 2$, and $T$ and $K+1 / 2$ are frequently used throughout this paper.

For the horizontal discretization, the Arakawa $\mathrm{C}$ grid is selected (Fig. 1b) because it has many advantages over other competing grids. One of the advantages is that it yields very good geostrophic adjustment characteristics. Also there are many established schemes for the C grid, which conserve or dissipate potential enstrophy while conserving energy. Among them, a fourth-order accurate scheme by Takano and Wurtele (1982) is selected. On the $\mathrm{C}$ grid shown in Fig. $1 \mathrm{~b}, h$ points contain $\theta, \pi_{\mathrm{qs}}$, $\delta \pi, \rho_{\mathrm{qs}}, T$, and $w$. The horizontal grid distance $d$ is uniform in both directions.

\section{b. Thermodynamic equation}

The first prediction made in the sequence of time integration steps is for the potential temperature $\left[\theta_{i+1 / 2, j+1 / 2, k}^{(n+1)}\right]$, where the superscript $n+1$ denotes the next time step. The thermodynamic equation in Eq. (2.5) on the grid shown in Fig. 1 is discretized using a flux-convergence inspired advective form in horizontal and following the second-order Adams-Bashforth scheme in time. Appendix A describes the discretization of the thermodynamic equation. A more detailed description of the discretization can be found in the supplementary material.

\section{c. Prediction of quasi-hydrostatic Exner pressure and quasi-hydrostatic density}

In this subsection, the diagnostic calculations of the quasi-hydrostatic Exner pressure and quasi-hydrostatic density in the discrete system are discussed. Since the prediction of the potential temperature for the next time step $n+1$ has already been carried out, the quasihydrostatic quantities can be diagnosed at time step $n+1$.

The discrete form of the vertical integral of Eq. (2.9a) from the surface to top can be written as

$$
\begin{aligned}
\left(\pi_{\mathrm{qs}}^{1 / \kappa}\right)_{i+1 / 2, j+1 / 2,1 / 2}^{(n+1)}= & \left(\pi_{\mathrm{qs}}^{1 / \kappa}\right)_{i+1 / 2, j+1 / 2, K+1 / 2}^{(n+1)} \\
& +\frac{g}{p_{00}} M_{i+1 / 2, j+1 / 2}^{(n+1)},
\end{aligned}
$$

where $p_{\mathrm{qs}}=p_{00} \pi_{\mathrm{qs}}^{1 / \kappa}$ is used. In Eq. (3.1),

$$
\begin{aligned}
M_{i+1 / 2, j+1 / 2}^{(n+1)}= & M_{i+1 / 2, j+1 / 2}^{(n)} \\
& -\delta t \sum_{k=1}^{K}\left[\nabla_{H} \cdot\left(\rho_{\mathrm{qs}} \mathbf{v}\right)_{k}\right]_{i+1 / 2, j+1 / 2}^{(n)}(\delta z)_{k},
\end{aligned}
$$

where $\delta t$ is the time step. Determination of $M$ from a prognostic equation is a deviation from Arakawa and Konor (2009). This choice enhanced the dynamical core's stability while maintaining the quality of solutions. In Eq. (3.2), the horizontal mass convergence $\left[\nabla_{H} \cdot\left(\rho_{\mathrm{qs}} \mathbf{v}\right)_{k}\right]_{i+1 / 2, j+1 / 2}^{(n)}$ is determined using a third-order scheme (see the supplementary material) and the boundary conditions in Eq. (2.4) are used. The discrete form of the vertical integral of Eq. (2.9b) from the top to surface yields $\left(\pi_{\mathrm{qS}}\right)_{i+1 / 2, j+1 / 2, K+1 / 2}^{(n+1)}=\left(\pi_{\mathrm{qs}}\right)_{i+1 / 2, j+1 / 2,1 / 2}^{(n+1)}-\sum_{k=1}^{K} g(\delta z)_{k} /$ $c_{p} \theta_{i+1 / 2, j+1 / 2, k}^{(n+1)}$. By eliminating $\left(\pi_{\mathrm{qS}}\right)_{i+1 / 2, j+1 / 2, K+1 / 2}^{(n+1)}$ between this equation and Eq. (3.1), we obtain an equation for the surface quasi-hydrostatic Exner pressure:

$$
\begin{aligned}
\left(\pi_{\mathrm{qs}}^{1 / \kappa}\right)_{i+1 / 2, j+1 / 2,1 / 2}^{(n+1)}= & {\left[\left(\pi_{\mathrm{qs}}\right)_{i+1 / 2, j+1 / 2,1 / 2}^{(n+1)}\right.} \\
& \left.-\frac{g}{c_{p}} \sum_{k=1}^{K} \frac{(\delta z)_{k}}{\theta_{i+1 / 2, j+1 / 2, k}^{(n+1)}}\right]^{1 / \kappa} \\
& +\frac{g}{p_{00}} M_{i+1 / 2, j+1 / 2}^{(n+1)} .
\end{aligned}
$$

This is an implicit equation, which is solved iteratively. The iteration starts by using $\left(\pi_{\mathrm{qs}}\right)_{i+1 / 2, j+1 / 2, K+1 / 2}^{(n)}$ for $\left(\pi_{\mathrm{qs}}\right)_{i+1 / 2, j+1 / 2, K+1 / 2}^{(n+1)}$ on the right-hand side of Eq. (3.3) and continues with a few correction steps. The second and last terms on the right-hand side remain unchanged during the iterations. In the test simulations, five iteration steps were sufficient for convergence. The quasihydrostatic Exner pressure for the interior interfaces and the top are obtained from a discrete form of the vertical integral of Eq. (2.9b) upward from surface starting from $\left(\pi_{\mathrm{qS}}\right)_{i+1 / 2, j+1 / 2, K+1 / 2}^{(n+1)}$, which is determined by Eq. (3.3). Finally, the time discrete form of Eq. (2.13) is written as

$$
\left(\rho_{\mathrm{qs}}\right)_{i+1 / 2, j+1 / 2, k}^{(n+1)}=\frac{p_{00}\left[\pi_{\mathrm{qs}}^{(1-\kappa) / \kappa}\right]_{i+1 / 2, j+1 / 2, k}^{(n+1)}}{R \theta_{i+1 / 2, j+1 / 2, k}^{(n+1)}},
$$

where

$$
\left(\pi_{\mathrm{qs}}\right)_{i+1 / 2, j+1 / 2, k}^{(n+1)} \equiv\left[\frac{\kappa}{p_{00}} \frac{\left.\left(p_{\mathrm{qs}}\right)_{k-1 / 2}^{(n+1)}-\left(p_{\mathrm{qs}}\right)_{k+1 / 2}^{(n+1)}\right]_{k-1 / 2}^{(n+1)}-\left(\pi_{\mathrm{qs}}\right)_{k+1 / 2}^{(n+1)}}{[\kappa /(1-\kappa)]}\right]_{i+1 / 2, j+1 / 2}
$$

The derivation of Eq. (3.5) is described in appendix B. Here $\partial \rho_{\mathrm{qs}} / \partial t$ and $\partial^{2} \rho_{\mathrm{qs}} / \partial t^{2}$ used on the right-hand side of the continuity equation in Eq. (2.3) and the elliptic equation in Eq. (2.15), respectively, are determined from the predicted $\rho_{\mathrm{qs}}$ through 


$$
\begin{aligned}
\left(\frac{\partial \rho_{\mathrm{qs}}}{\partial t}\right)_{i+1 / 2, j+1 / 2, k}^{(n)} \equiv & {\left[\frac{4}{3} \frac{\left(\rho_{\mathrm{qs}}\right)^{(n+1)}-\left(\rho_{\mathrm{qs}}\right)^{(n)}}{\delta t}\right.} \\
& \left.-\frac{1}{3} \frac{\left(\rho_{\mathrm{qs}}\right)^{(n+1)}-\left(\rho_{\mathrm{qs}}\right)^{(n-1)}}{2 \delta t}\right]_{i+1 / 2, j+1 / 2, k}
\end{aligned}
$$

and the second time derivative of $\rho_{\mathrm{qs}}$ is temporally discretized as

$$
\begin{aligned}
\left(\frac{\partial^{2} \rho_{\mathrm{qs}}}{\partial t^{2}}\right)_{i+1 / 2, j+1 / 2, k}^{(n)} \equiv & \frac{1}{\delta t}\left[\left(\frac{\partial \rho_{\mathrm{qs}}}{\partial t}\right)^{(n)}\right. \\
& \left.-\left(\frac{\partial \rho_{\mathrm{qs}}}{\partial t}\right)^{(n-1)}\right]_{i+1 / 2, j+1 / 2, k}
\end{aligned}
$$

These definitions differ from those suggested by Arakawa and Konor (2009). While carrying out the test simulations, it was found that the model's stability depends on how the first and second time derivatives are determined. It turns out that the use of information from three consecutive time levels to determine the first derivative greatly enhances stability. On the basis of experiments with several formulations, the form in Eq. (3.6a) has been decided. The solutions were stable for a range of coefficient pairs in Eq. (3.6a). We have selected the pair $(4 / 3)$ and $(-1 / 3)$ for simplicity.

\section{d. Determination of the nonhydrostatic Exner pressure and other nonhydrostatic quantities}

We follow the same multistep procedure used in the derivation of the elliptic continuous equation in Eq. (2.15) to derive the discrete version of the elliptic equation. The discrete versions of the continuity and momentum equations are used. The resulting discrete elliptic equation is used to determine $d p$ for the time step $n+1$. The supplementary material discusses the derivation of the discrete elliptic equation in detail.

There is a difference between the elliptic equations in the unified and the anelastic (or original pseudoincompressible) systems. In the unified system, the elliptic equation determines $\delta \pi$, which is the deviation of $\pi$ from the locally predicted $\pi_{\mathrm{qs}}$ while, in the anelastic system it determines $\pi^{\prime}$, that is the deviation from a horizontally uniform fixed mean (or reference) state $\bar{\pi}(z)$. In the large-scale or global applications, the elliptic solver in the unified system needs to do much less work than that in the anelastic system because the spatial and temporal fluctuations of $\delta \pi$ are, in general, much smaller than those of $\pi^{\prime}$. For the solution of the elliptic equation, we use an iterative method described in the supplementary material. In the extratropical cyclogenesis simulations with low $(100 \mathrm{~km})$ and moderate $(50 \mathrm{~km})$ horizontal resolutions presented here, 15 iterations were enough for convergence. No stopping criterion is used in the solver. The iteration numbers given here are empirically determined from performing multiple simulations with different number of iterations and observing the convergence of solutions.

The only nonhydrostatic quantity needed in the dynamical core is $\delta \pi$, which is used in the pressure gradient force term. However, the physical parameterizations used in comprehensive atmosphere models need the nonhydrostatic pressure, temperature, and density for various calculations. The relations that determine these quantities from the nonhydrostatic Exner pressure can be found in the supplementary material.

\section{e. The horizontal momentum equation}

The $u$ and $v$ components of the (vector) horizontal momentum equation in Eq. (2.6) are spatially and temporally discretized as

$$
u_{i, j+1 / 2, k}^{(n+1)}=u_{i, j+1 / 2, k}^{(n)}+\delta t\left(G_{u}\right)_{i, j+1 / 2, k}^{(n+1 / 2)}-\delta t c_{p} \theta_{i, j+1 / 2, k}^{(n+1)}\left[\frac{\left(\pi_{\mathrm{qs}}+\delta \pi\right)_{i+1 / 2}^{(n+1)}-\left(\pi_{\mathrm{qs}}+\delta \pi\right)_{i-1 / 2}^{(n+1)}}{d}\right]_{j+1 / 2, k}+\delta t\left(F_{u}\right)_{i, j+1 / 2, k}^{(n+1)}
$$

and

$$
v_{i+1 / 2, j, k}^{(n+1)}=v_{i+1 / 2, j, k}^{(n)}+\delta t\left(G_{v}\right)_{i+1 / 2, j, k}^{(n+1 / 2)}-\delta t c_{p} \theta_{i+1 / 2, j, k}^{(n+1)}\left[\frac{\left(\pi_{\mathrm{qs}}+\delta \pi\right)_{j+1 / 2}^{(n+1)}-\left(\pi_{\mathrm{qs}}+\delta \pi\right)_{j-1 / 2}^{(n+1)}}{d}\right]_{i+1 / 2, k}+\delta t\left(F_{v}\right)_{i+1 / 2, j, k}^{(n+1)}
$$

respectively. In Eqs. (3.7a) and (3.7b), the horizontal and vertical advective tendencies $\left(G_{u}\right)_{i, j+1 / 2, k}^{(n+1 / 2)}$ and $\left(G_{v}\right)_{i+1 / 2, j, k}^{(n+1 / 2)}$ are temporally discretized following the second-order Adams-Bashforth scheme. The horizontal advective terms 
are based on a fourth-order potential enstrophy dissipating scheme described by the supplementary material. For the discretization of the pressure gradient force terms, the economical implicit time integration scheme is used. Note that $\left(\pi_{\mathrm{qS}}\right)_{i+1 / 2, j+1 / 2, k}^{(n+1)}$ and $\delta \pi_{i+1 / 2, j+1 / 2, k}^{(n+1)}$ are determined from Eq. (3.5) and the elliptic equation, respectively. In Eqs. (3.7a) and (3.7b), the superscript $(n+1)$ in the friction (and diffusion) terms denotes that their contributions are calculated and added after the values of velocities are updated with the contributions from all other tendencies.

\section{$f$. The continuity equation and prediction of vertical mass flux and vertical velocity}

The continuity equation given by Eq. (2.3) is first temporally and spatially discretized and then multiplied by $(\delta z)_{k}$ to obtain the equation that is used to determine the vertical mass flux as

$$
\begin{aligned}
\left(\rho_{\mathrm{qs}} w\right)_{i+1 / 2, j+1 / 2, k+1 / 2}^{(n+1)}= & \left(\rho_{\mathrm{qs}} w\right)_{i+1 / 2, j+1 / 2, k-1 / 2}^{(n+1)} \\
& -(\delta z)_{k}\left[\mathbf{\nabla}_{H} \cdot\left(\rho_{\mathrm{qs}} \mathbf{v}\right)_{k}\right]_{i+1 / 2, j+1 / 2}^{(n+1)} \\
& -(\delta z)_{k}\left(\frac{\partial \rho_{\mathrm{qs}}}{\partial t}\right)_{i+1 / 2, j+1 / 2, k}^{(n)}
\end{aligned}
$$

Following the boundary conditions in Eq. (2.4), the vertical mass flux at surface is prescribed as

$$
\left(\rho_{\mathrm{qs}} w\right)_{i+1 / 2, j+1 / 2,1 / 2}^{(n+1)}=0 .
$$

Note that, $\left[\nabla_{H} \cdot\left(\rho_{\mathrm{qs}} \mathbf{v}\right)_{k}\right]_{i+1 / 2, j+1 / 2}^{(n+1)}$ is determined after the prediction of velocities are completed by using $\left(\rho_{\mathrm{qs}}\right)_{i+1 / 2, j+1 / 2, k}^{(n+1)}$. Finally, the vertical velocity is determined from

$w_{i+1 / 2, j+1 / 2, k+1 / 2}^{(n+1)} \equiv\left[\left(\rho_{\mathrm{qs}} w\right)^{(n+1)} /\left(\rho_{\mathrm{qs}}\right)^{(n+1)}\right]_{i+1 / 2, j+1 / 2, k+1 / 2}$,

where $\left(\rho_{\mathrm{qs}}\right)_{i+1 / 2, j+1 / 2, k+1 / 2}^{(n+1)}$ is obtained through a vertical interpolation.

\section{g. Summary of the integration procedure}

The unified system splits the scalar quantities, such as density, Exner pressure, and temperature, into their quasi-hydrostatic and nonhydrostatic components, and predicts these components separately. This structure makes the time integration procedure different than that in the conventional models. Figure 2 presents a flowchart of the integration procedure used in the discrete model. The integration starts with the prediction of $\theta$ for the next time step using the information from the

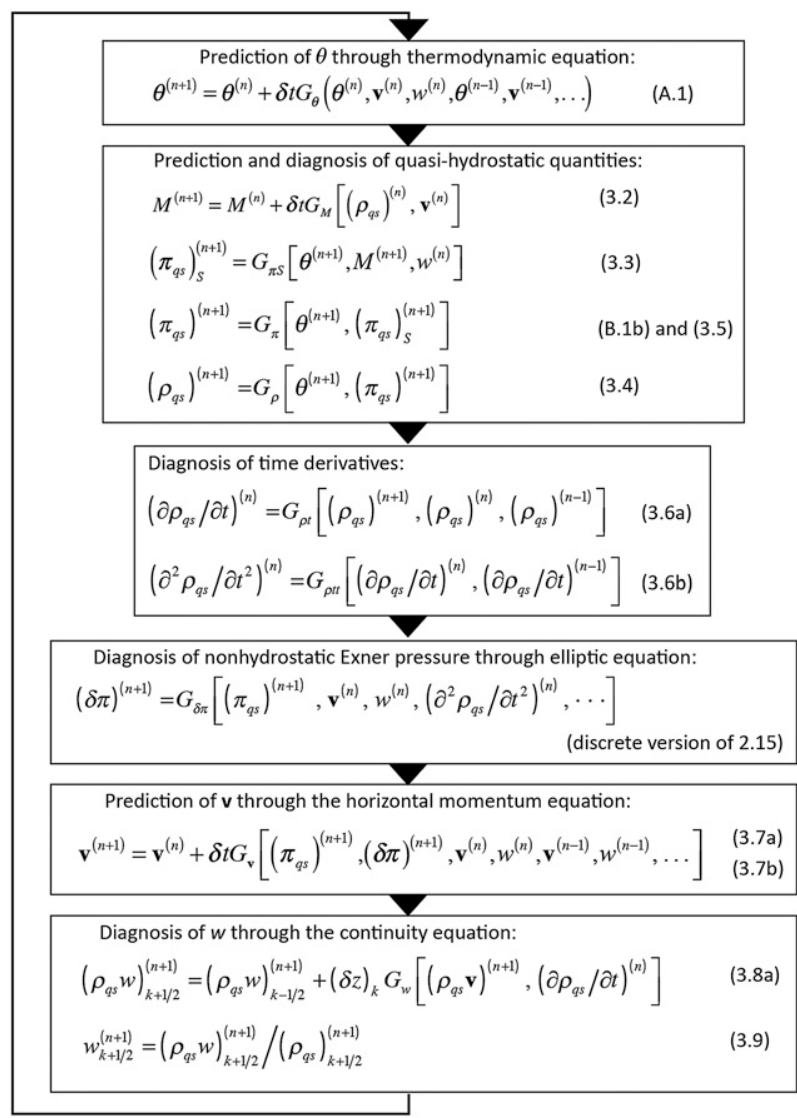

FIG. 2. A flowchart showing the integration procedure with the unified model. Function $G$ is used to shorten the equations. The exact form of the equations can be found in the text through indicated equation numbers.

current time step. Next comes the prediction of $\left(\pi_{\mathrm{qs}}\right)_{S}$ from the predicted $\theta$. Then, $\pi_{\mathrm{qs}}$ is determined for interior points using $\left(\pi_{\mathrm{qs}}\right)_{S}$ and $\theta$. Next, the quasi-hydrostatic density $\rho_{\mathrm{qs}}$ is predicted for the next time step. After determining $\partial \rho_{\mathrm{qs}} / \partial t$ and $\partial^{2} \rho_{\mathrm{qs}} / \partial t^{2}$, the nonhydrostatic Exner pressure is obtained for the next step by solving the elliptic equation. Next, the horizontal component of momentum is predicted using already predicted $\pi_{\mathrm{qs}}+\delta \pi$ in the pressure gradient force. Finally, the vertical mass flux and vertical velocity are obtained for the next time step by vertically integrating the continuity equation. Note that if $w^{(n)}$ is not available at the beginning, it can be determined by using $\mathbf{v}^{(n)}$ in the discrete continuity equation in Eq. (3.17) by assuming $\left(\partial \rho_{\mathrm{qs}} / \partial t\right)^{(n)}=0$.

\section{Test simulations with the dynamical core}

To demonstrate the performance of the dynamical core in simulating different scales of atmospheric motion, the results from cyclone- and cloud-scale simulations are presented in this section. 


\section{a. Cyclone-scale simulations}

\section{1) Simulations of IDEALIZED EXTRATROPiCAL CYCLOGENESIS STARTING FROM RANDOM PERTURBATIONS}

Simulations of idealized extratropical cyclogenesis on a midlatitude $b$ plane are used to evaluate the performance of the dynamical core based on the unified system. The purpose of this simulation is to evaluate the model's behavior under noisy conditions. We performed a set of simulations that start by adding small-amplitude random perturbations $( \pm 1 \mathrm{~K})$ on the initial zonally uniform potential temperature, as in Arakawa and Konor (1996) and Konor and Arakawa (1997).

We limit the physical parameterizations used in these simulations to the surface friction prescribed by $\left(\mathbf{F}_{H}\right)_{S} \equiv$ $-C_{D}\left|\mathbf{v}_{S}\right| \mathbf{v}_{S}$ with $C_{D}=0.5 \times 10^{-3}$ (applied to the lowest layer) and fourth-order horizontal diffusions of potential temperature and horizontal momentum with the coefficient $\kappa_{H}=(d / 100)^{4} \times 10^{13} \mathrm{~m}^{4} \mathrm{~s}^{-1}$, where $d$ is the horizontal grid distance used in kilometers. Note that the diffusion coefficient used here corresponds to the weak diffusion used by Arakawa and Konor (1996) for $d=100 \mathrm{~km}$. The coefficient of the potential enstrophy dissipation $\tau_{\text {ed }}$ used is $10 \times(d / 100 \mathrm{~km}) \mathrm{s}$ below $8 \mathrm{~km}$. Above this height, it increases linearly with height and becomes $30 \times(d / 100 \mathrm{~km}) \mathrm{s}$ at the top. The horizontal domain is a channel $5000 \mathrm{~km}$ long and $13000 \mathrm{~km}$ wide, and the depth of the domain is $18 \mathrm{~km}$. The horizontal channel is bounded by flux-free walls at the northern and southern boundaries. In the zonal direction, cyclic conditions are used. There are 45 equally spaced layers in the vertical, which corresponds to 400-m vertical grid spacing. For the $100-\mathrm{km}$ horizontal resolution, the solutions are stable with a 2-min time step. In the other simulations, the time step per horizontal grid distance is determined from $2 \times(d / 100 \mathrm{~km}) \mathrm{min}$.

The zonally uniform component of the initial flow is shown in Fig. 3. A zonal jet with the maximum speed of $58 \mathrm{~m} \mathrm{~s}^{-1}$ and zero speed at the surface and upper boundary is prescribed. The zonally uniform component of the potential temperature is determined by assuming geostrophic balance and prescribing the square of the Brunt-Väisäla frequency at the southern boundary of the domain as $N^{2}=0.0002 \mathrm{~s}^{-2}$ and $N^{2}=0.0005 \mathrm{~s}^{-2}$ for the simulated troposphere (below $10 \mathrm{~km}$ ) and stratosphere, respectively. The channel is assumed to be centered at $45^{\circ} \mathrm{N}$, which yields $f_{0}=1.03 \times 10^{-4} \mathrm{~s}^{-1}$ and $\beta=1.62 \times 10^{-11} \mathrm{~m}^{-1} \mathrm{~s}^{-1}$. The simulations are started from a small-amplitude random potential temperature perturbation (maximum $\pm 1 \mathrm{~K}$ ) superimposed on the otherwise zonally uniform potential temperature field.

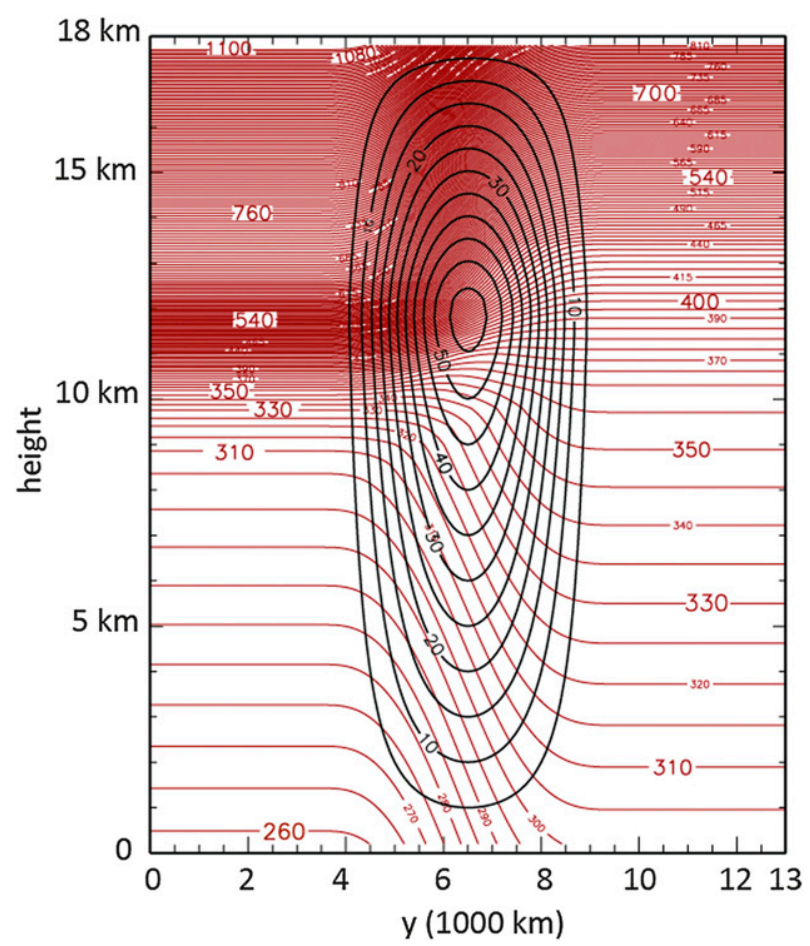

FIG. 3. The zonal velocity ( $\mathrm{m} \mathrm{s}^{-1}$, thick lines) and the potential temperature $(\mathrm{K}$, thin lines) of the zonal component of the initial flow. Lowest contour value for the potential temperature and velocity are $260 \mathrm{~K}$ and $5 \mathrm{~m} \mathrm{~s}^{-1}$, respectively. The contour intervals are $5 \mathrm{~K}$ and $5 \mathrm{~m} \mathrm{~s}^{-1}$, respectively.

The amplitude of perturbation is largest in the lowest layer, decreases linearly with height, and vanishes at and above $8 \mathrm{~km}$.

Four simulations are made using the horizontal grid spacing of $100,50,25$, and $12.5 \mathrm{~km}$ with the same vertical resolution. Empirical tests show that the elliptic solver needs 10, 15, 25, and 50 iterations for convergence with these resolutions, respectively. The initial random potential temperature perturbation is originally prescribed on the $100-\mathrm{km}$ grid and interpolated to the higherresolution grids so that the initial conditions are virtually identical for all the simulations regardless of the horizontal resolutions. The integrations are made for 20 simulated days.

Figure 4 shows surface pressure and surface potential temperature for days 15 and 17 of the two simulations with $100-$ and 12.5 -km horizontal grid spacings. The fields from the $12.5-\mathrm{km}$ simulation are written out and plotted using the $25-\mathrm{km}$ grid to reduce the size of the datasets. This can be justified for this case because the $25-\mathrm{km}$ grid spacing is already high enough to see the structure of the solution, and no detailed dynamical analysis is performed using the data. At day 15 (Fig. 4, top panel), a well-developed primary extratropical cyclone is apparent at the surface fields 


\section{Surface pressure $\left(p=p_{q s}+\delta p, \mathrm{mb}\right)$ and surface potential temperature (K)}
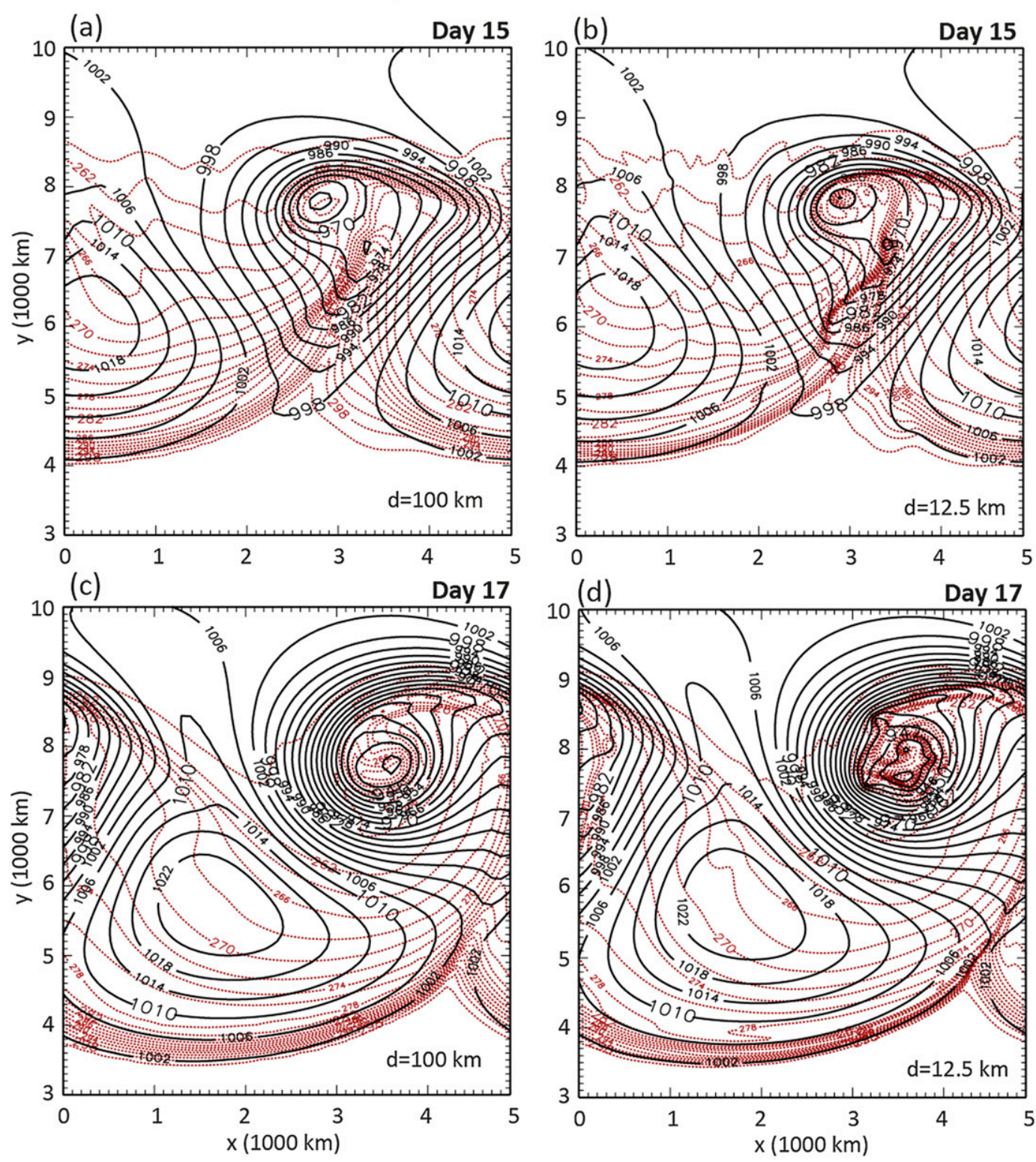

FIG. 4. Surface pressure (total of quasi-hydrostatic and nonhydrostatic pressures, mb, thick black lines) and surface potential temperature (K, dotted red lines) from (a) 100-km resolution run at day 15 , (b) 12.5-km resolution run at day 15, (c) $100-\mathrm{km}$ resolution run at day 17 , and (d) $12.5-\mathrm{km}$ resolution run at day 17 . The contour interval for the pressure and potential temperature are $4 \mathrm{mb}$ and $2 \mathrm{~K}$, respectively.

in both simulations. While the depth of the cyclone is nearly the same for both resolutions at day 15 , the intensities of surface fronts are stronger in the $12.5-\mathrm{km}$ simulation that that in the $100-\mathrm{km}$ simulation. The cold, warm, and bent-back warm fronts are already pronounced at this time. A secondary front has also developed, in association with an intense trough behind the primary cold front. At day 17, the cyclogenesis reaches its most mature stage (Figs. 4c,d), in which strong cyclonic surface wind wraps the bent-back warm front around the cyclone center. Secondary cyclones resulting from shearing instability are clearly visible around the primary cyclone center in the high-resolution simulation (Fig. 4d). Figure 5 presents close-up of the surface vorticity associated with these secondary cyclones at day 17 in the $12.5-\mathrm{km}$ simulation. The development of the intense cyclonic vortices in a highhorizontal shear zone can be clearly seen in the vorticity field. After day 17, the deepening of surface cyclones 


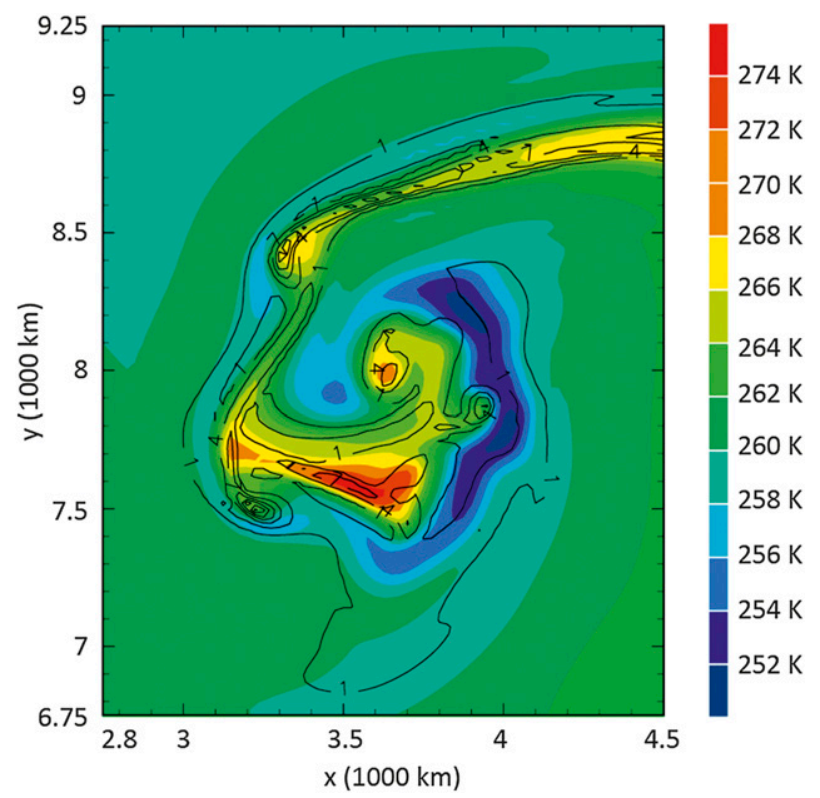

FIG. 5. A close-up of surface vorticity $\left(10^{-4} \mathrm{~s}^{-1}\right.$, solid lines $)$ and surface potential temperature ( $\mathrm{K}$, colored shading) focusing on the low pressure center at day 17 of the high-resolution run $(d=$ $12.5 \mathrm{~km})$. The contour interval of vorticity is $3 \times 10^{-4} \mathrm{~s}^{-1}$.

stops, and the solutions gradually become disorganized. Although it is not shown here (see the supplementary material), these simulations also show realistic upperlevel frontogenesis, jet-streak dynamics, and tropopause folding. Many aspects of these simulations qualitatively agree with those of Shapiro (1975), Newton and Trevisan (1984), Konor and Arakawa (1997), and many others.

\section{2) STARTING FROM SMOOTH LARGE-SCALE PERTURBATIONS}

The purpose of this test is to evaluate the performance of the model for the extratropical cyclogenesis case suggested by Ullrich and Jablonowski (2012). The horizontal resolution is $100 \mathrm{~km}$, and there are 30 equally spaced layers in the vertical between the surface and $30 \mathrm{~km}$. In Fig. 6, we show the results from the $\beta$-plane case for days 10 and 12 of the simulation. The unified dynamical core and Ullrich and Jablonowski (2012) simulations closely resemble each other. The simulation with the unified model shows slightly deeper cyclones, stronger fronts, and sharper isobar kinks across the fronts.

\section{b. Cloud-scale simulations}

\section{1) WARM BUBbLE SIMULATIONS}

A two-dimensional version of the dynamical core used for the simulation of extratropical cyclogenesis has been applied to the warm bubble simulations without rotation. The initial potential temperature perturbation used in these simulations is obtained from

$$
\theta^{\prime}(x, y, z) \equiv \operatorname{Max}[\Theta(1-R), 0],
$$

where $\Theta$ is the maximum of potential temperature perturbation and $R \equiv\left(x-x_{0}\right)^{2} / L_{x}^{2}+\left(z-z_{0}\right)^{2} / L_{z}^{2}$, where $\left(x_{0}, z_{0}\right)$ is the center point of the oval perturbation, and $L_{x}$ and $L_{z}$ are horizontal and vertical lengths, respectively, of the perturbation. The initial perturbation described by Eq. (4.1) used in the two separate simulations,
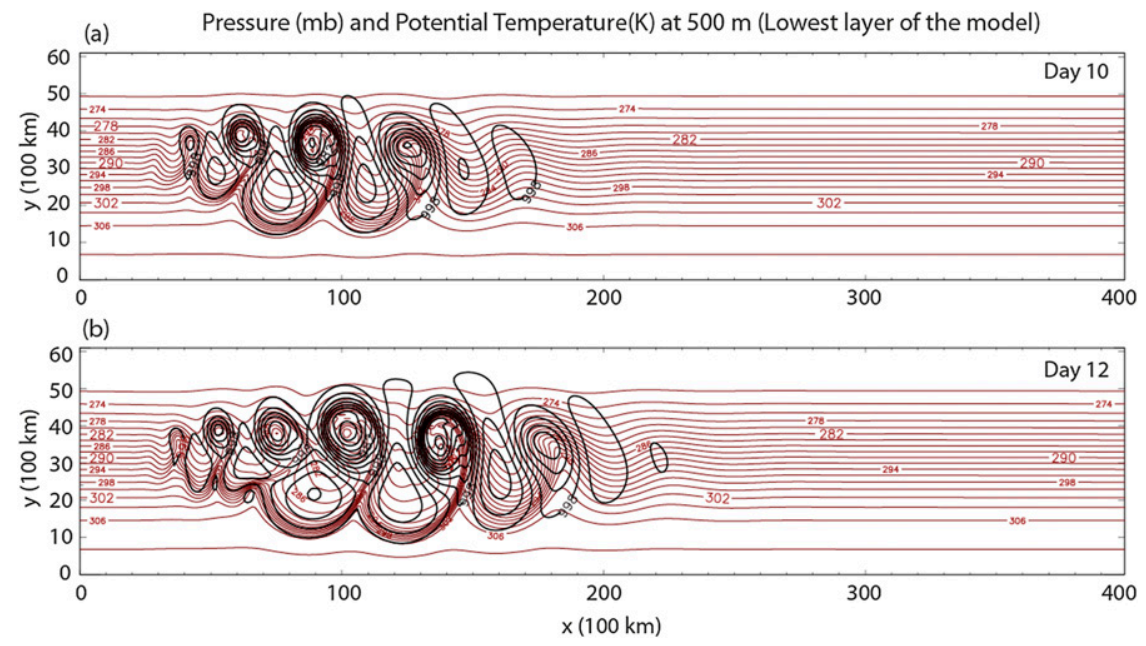

FIG. 6. The pressure (total of quasi-hydrostatic and nonhydrostatic pressures, mb, thick black lines) and potential temperature (K, red thin lines) for 500-m height surface (lowest layer of the model) at (a) day 10 and (b) day 12. One of the contour values for the pressure is $998 \mathrm{mb}$. The contour intervals for the pressure and potential temperature are $4 \mathrm{mb}$ and $2 \mathrm{~K}$, respectively. 
(a)

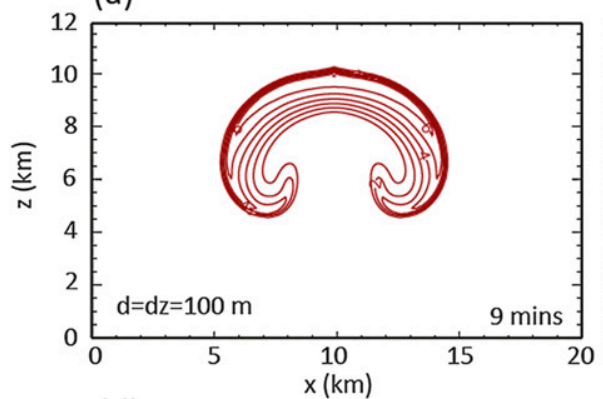

(d)

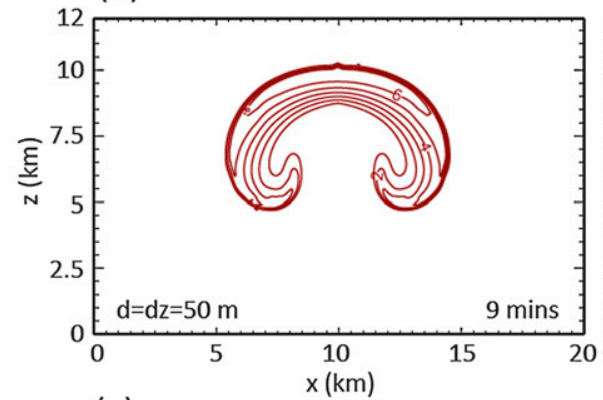

(g)

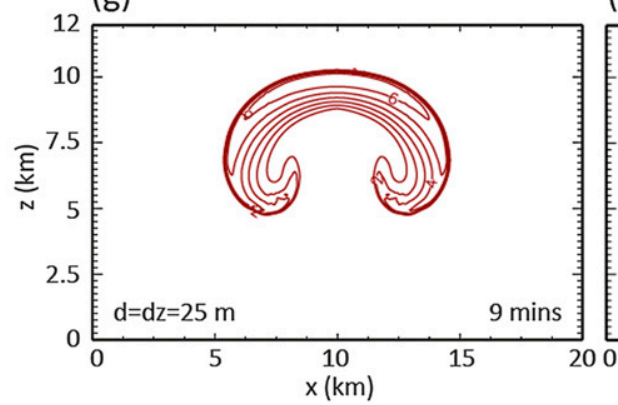

(b)

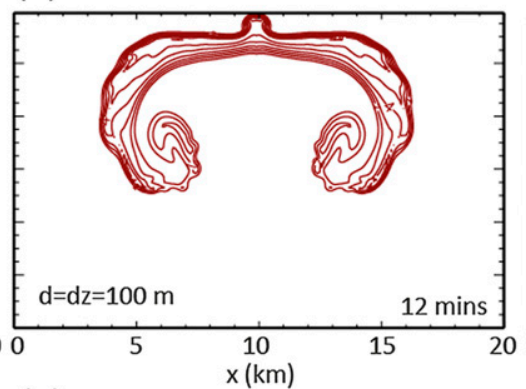

(e)

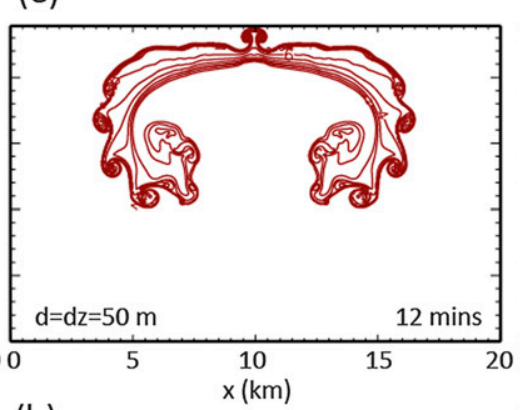

(h)

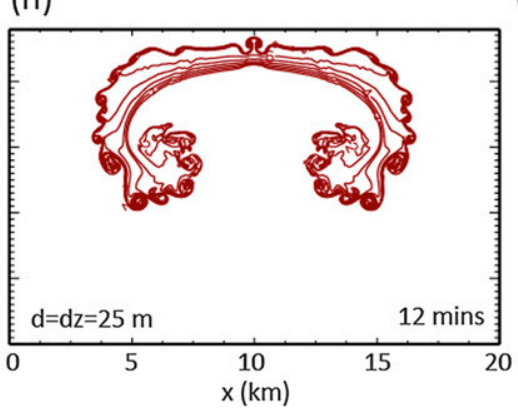

(c)

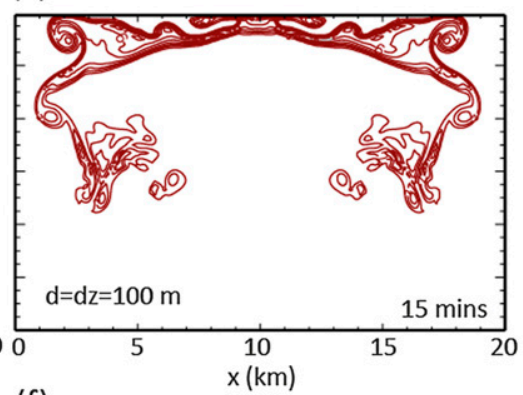

(f)

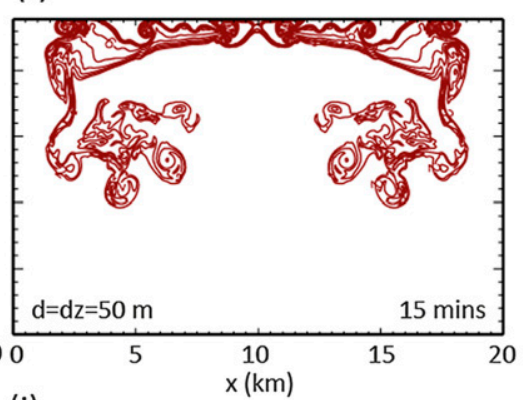

(i)

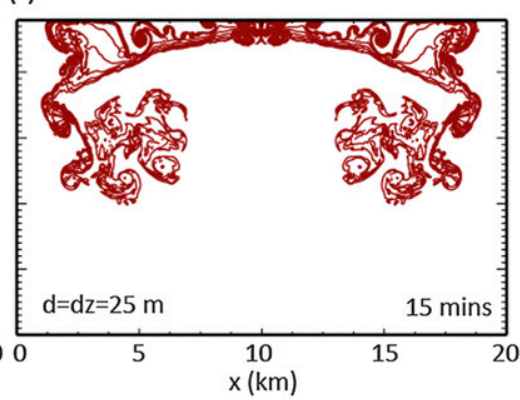

FIG. 7. The potential temperature perturbation (K) from the (top) 100-m, (middle) 50-m, and (bottom) 25-m simulations at (a),(d),(g) $9 \mathrm{~min}$; (b),(e),(h) $12 \mathrm{~min}$; and (c),(f),(g) $15 \mathrm{~min}$. The lowest contour value and contour interval are both $1 \mathrm{~K}$.

which are discussed next, with different shapes and maximums.

\section{(i) Warm bubble in a neutrally stratified environment}

In the first simulation, the environment potential temperature is set to $300 \mathrm{~K}$ throughout the domain. An initial perturbation given by Eq. (4.1) with $\Theta=6.6 \mathrm{~K}$, $L_{x}=L_{z}=2500 \mathrm{~m}, z_{0}=2750 \mathrm{~m}$, and $x_{0}$ chosen as the center of the horizontal domain is superimposed on this environment following Mendez-Nunez and Carroll (1994), Gallus and Rancic (1996), and Janjic et al. (2001). We used a fourth-order diffusion of potential temperature and momentum with a small coefficient $\left(5 \mathrm{~m}^{4} \mathrm{~s}^{-1}\right)$. This diffusion can be omitted all together without any visible change in the results. The horizontal domain of the model is $20 \mathrm{~km}$ wide and the lateral boundary conditions are cyclic. The vertical domain is
$12 \mathrm{~km}$ deep. Three simulations are performed with 100-, 50-, and 25-m horizontal and vertical grid spacings, using time steps of $0.5,0.3$, and $0.25 \mathrm{~s}$, respectively. The elliptic solver used 100 iterations. The number of iterations was determined through empirical convergence tests.

Figure 7 shows the simulated potential temperature perturbation at 9,12 , and $15 \mathrm{~min}$ from the three simulations. At minute 9, the bubble rises with a strong center updraft while a strong temperature gradient forms on the outer shell of the bubble (Figs. 11a,d,g). At this stage, the impact of resolution on the solutions appears negligible. At minute 12, the upper tip of the rising bubble reaches the upper boundary in all simulations. On the outer shell of the rising bubble, small vortices develop due to a shearing instability in the 50 - and $25-\mathrm{m}$ simulations (Figs. 11e and 11h, respectively). In the 100-m simulation, there are again signs of shearing instability, but the vortices are not fully formed (Fig. 11a). 
(a)

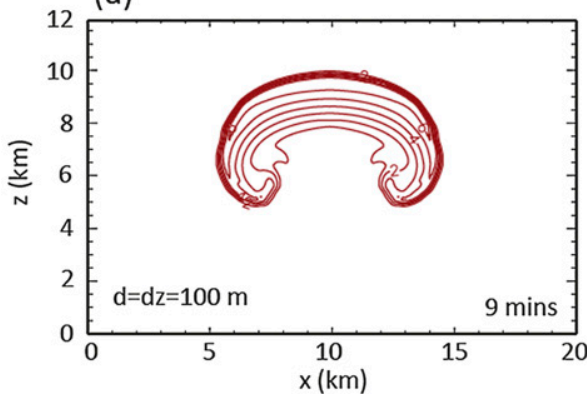

(d)

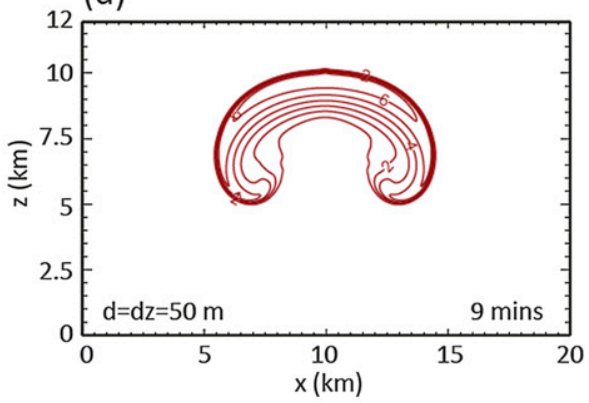

(g)

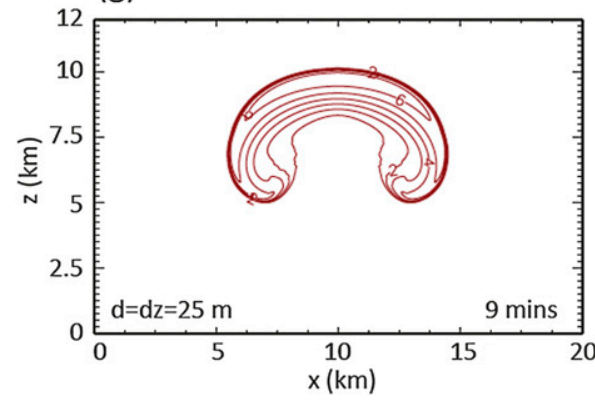

(b)

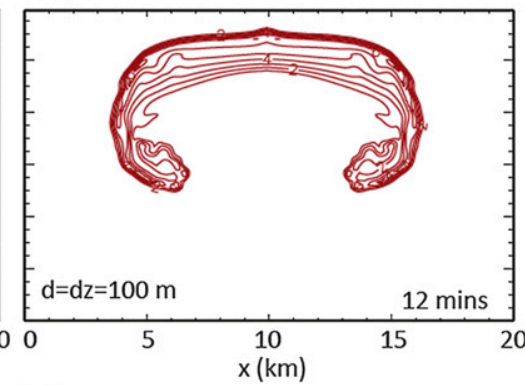

(e)

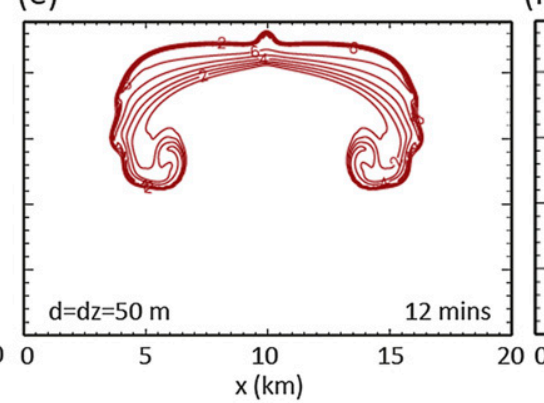

(h)

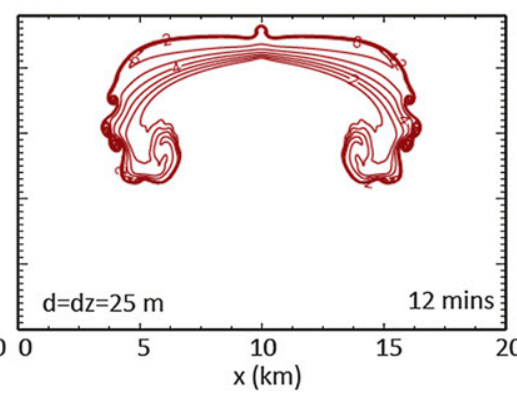

(c)

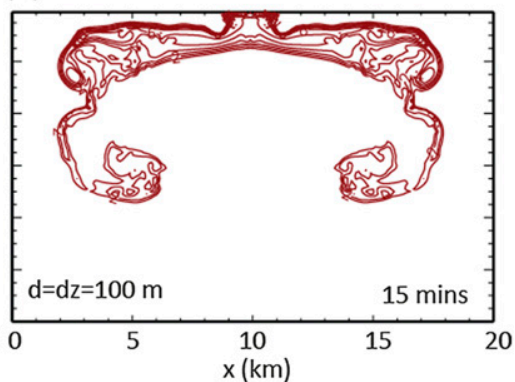

(f)

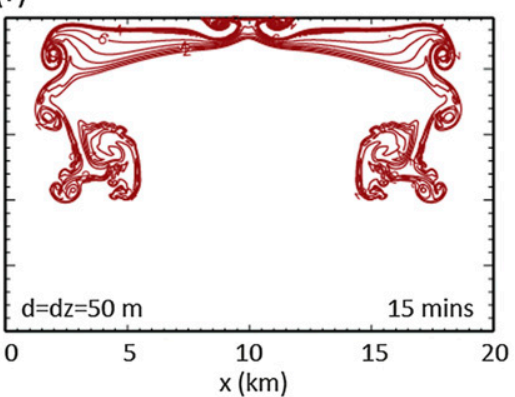

(i)

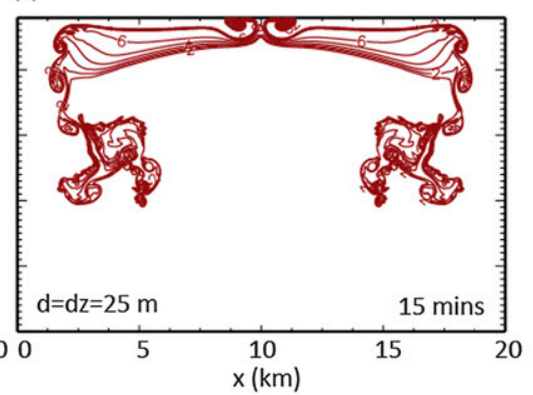

FIG. 8. As in Fig. 7, but from the fully compressible model.

These results agree overall with those discussed in the literature (e.g., Grabowski and Clark 1991; Smolarkiewicz and Pudykiewicz 1992; Robert 1993; Kurowski et al. 2013; and many others). Once the bubble reaches the upper boundary it spreads out as shown in Figs. 11c,f,i. In the wake of the bubble, air from the bubble mixes with the environment. It appears that the intensity of this mixing increases with the resolution.

To examine how the simulations with the unified equations model compare to those obtained by a fully compressible model, we constructed a fully compressible model by using the general structure and the numerical schemes of the unified model. In the fully compressible model, the mass-weighted vertical velocity and density are predicted from the vertical momentum and continuity equations, respectively. The Exner pressure is obtained from the predicted potential temperature and density using the state equation. A second-order Adams-Bashforth time integration scheme is used in both the vertical momentum and continuity equations with very small time steps that maintain the stability. No explicit diffusion is added in these simulations. Figure 8 shows the results from the fully compressible model. Overall, these results agree well with those of the unified model. However, there are differences in the development of the small vortices resulted from the shearing instability. These small vortices are weaker in the fully compressible model results than in the unified model results.

An upgraded version of the fully compressible model has also been constructed by increasing the accuracy of the selected schemes. The horizontal mass fluxes were upgraded to a fifth-order scheme, from third order in the original model; the horizontal potential temperature fluxes are also upgraded in the same way; and the vertical momentum advection was calculated following a fourth-order scheme instead of second order. Figure 9 shows the results obtained by the upgraded fully compressible model. If these results are compared to those 
(a)

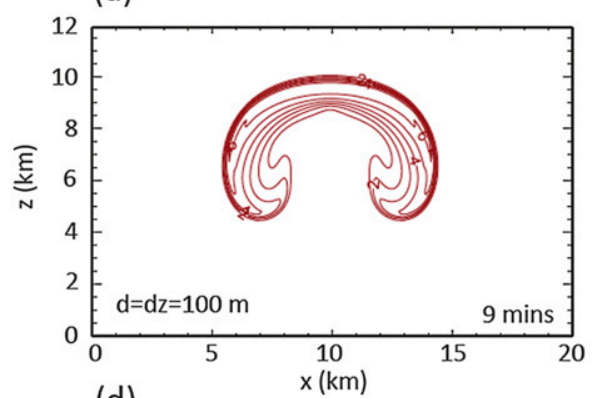

(d)

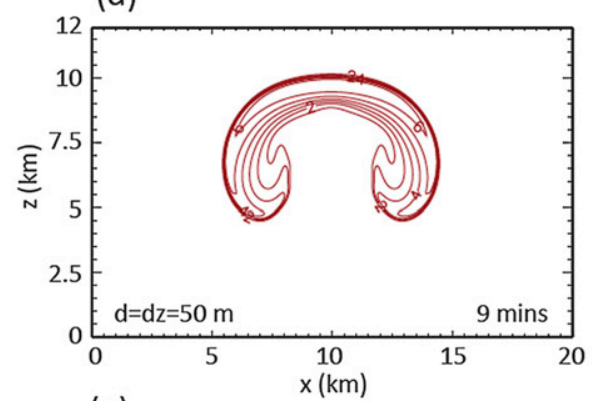

(g)

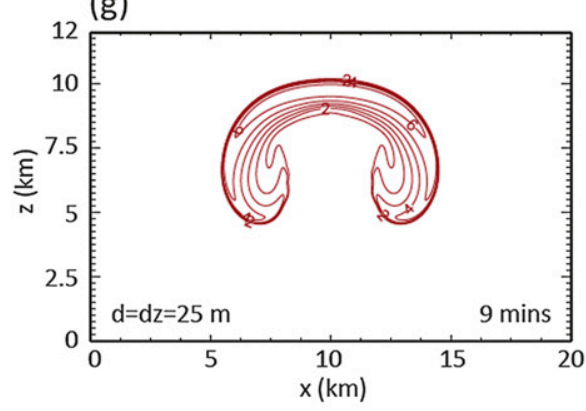

(b)

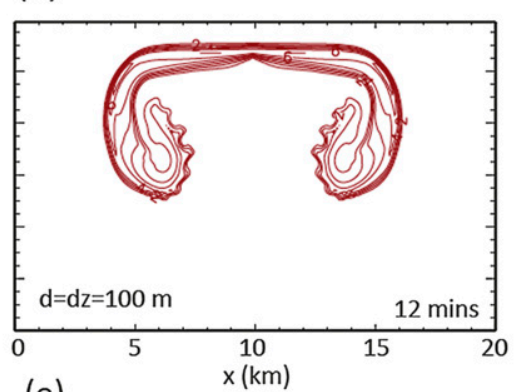

(e)

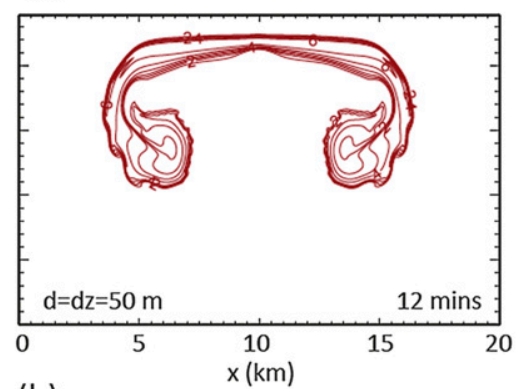

(h)

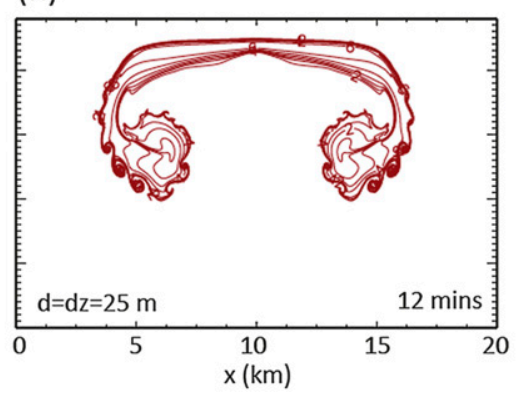

(c)

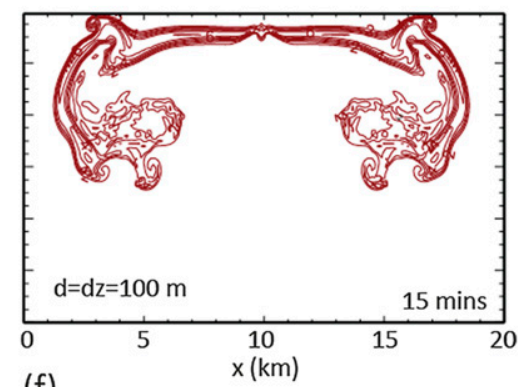

(f)

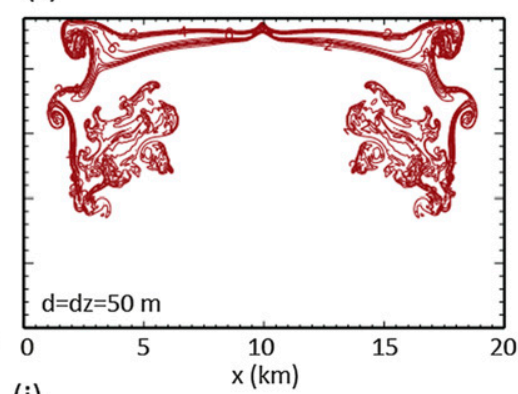

(i)

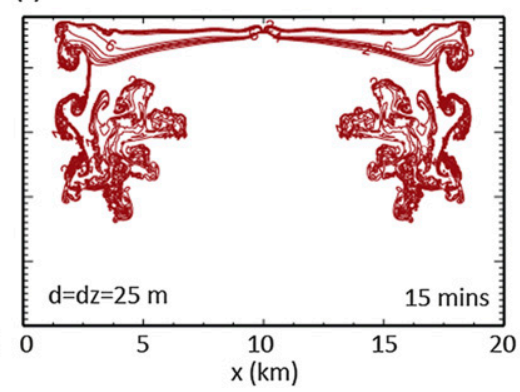

FIG. 9. As in Fig. 7, but from the fully compressible model with the higher-order advection schemes.

shown in Fig. 8, there is definitely an improvement in capturing the small-scale features earlier in the integrations. At this point, we do not exactly know the source of the differences between the unified and the fully compressible models in simulating these smallscale features. The test with the more accurate schemes suggests that the fully compressible model requires higher-order and better schemes to capture the shearing instability. We further investigate the differences of solutions between the different dynamical cores.

\section{(ii) Warm bubble in a neutral environment topped} with a stable layer

The purpose of this simulation is to evaluate the unified dynamical core's behavior in a stable stratospherelike environment. In this simulation, the environment is modified by adding a stable layer with $N^{2}=0.0003 \mathrm{~s}^{-2}$ above $5000 \mathrm{~m}$. The maximum of the potential temperature perturbation is set to $\Theta=6 \mathrm{~K}$ with $L_{x}=L_{z}=750 \mathrm{~m}$ and $z_{0}=3000 \mathrm{~m}$ in the formula given by Eq. (4.1). Figure 10 shows the initial perturbation and basic state used in these simulations. The horizontal domain of the models is $10 \mathrm{~km}$ wide and the model top is $60 \mathrm{~km}$ above the surface. The horizontal and vertical grid spacings are $50 \mathrm{~m}$ and the coefficient of the fourth-order diffusion is $\left(100 \mathrm{~m}^{4} \mathrm{~s}^{-1}\right)$. We have also performed these simulations with the fully compressible (version using the schemes of the unified dynamical core), anelastic, and pseudoincompressible dynamical cores for comparison purposes. The anelastic and pseudo-incompressible dynamical cores used in the comparison are based on the Lipps and Hemler (1982) and Durran (1989) systems, respectively. The anelastic and pseudo-incompressible dynamical cores were also constructed using (as far as possible) the schemes of the unified dynamical core.

Figure 11 shows the simulated potential temperature perturbation at $5,15,30$, and $60 \mathrm{~min}$ in to the run, as simulated with the unified, fully compressible, anelastic, 

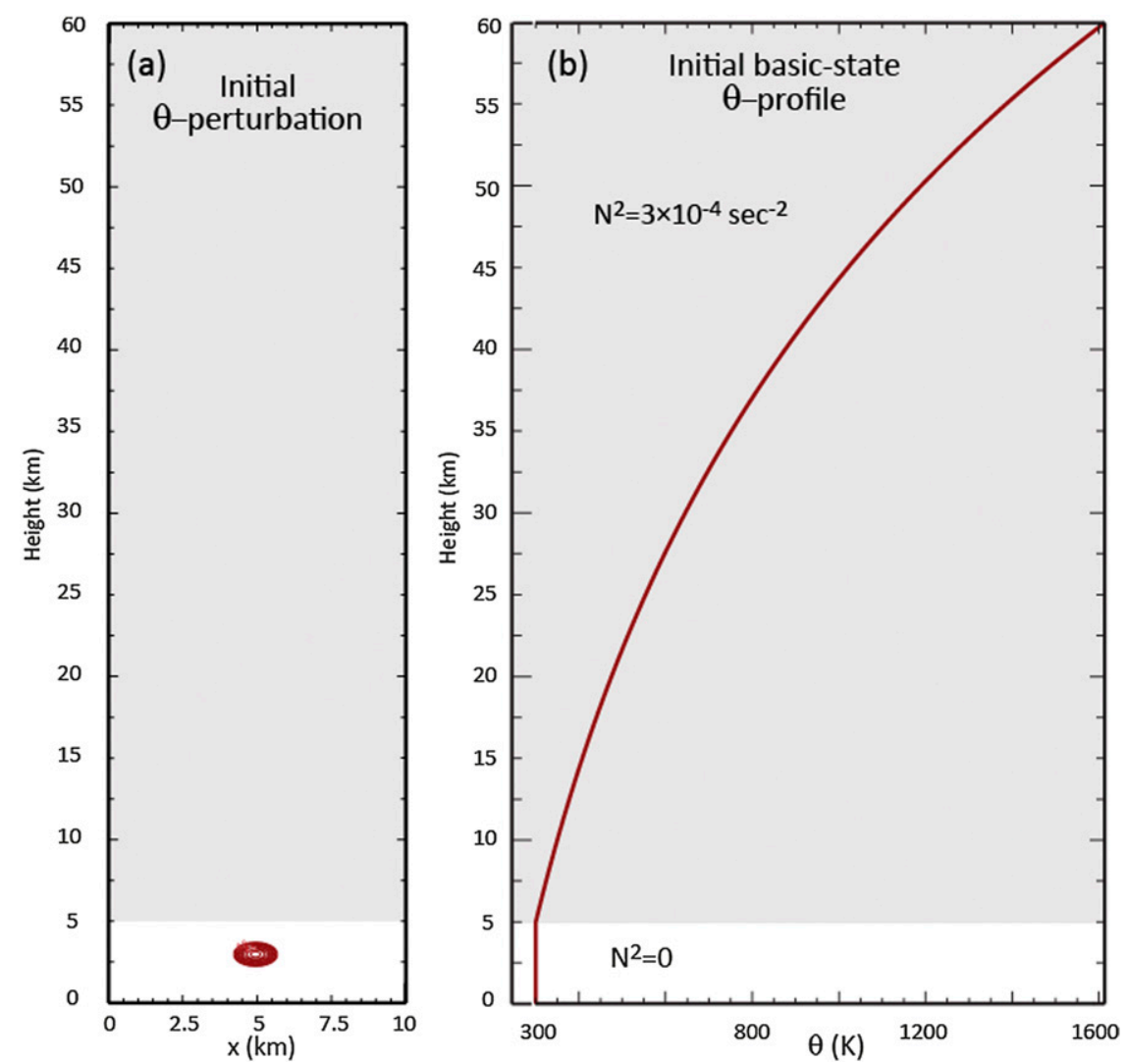

FIG. 10. (a) The initial potential temperature perturbation (K) and (b) initial basic-state potential temperature profile of the warm bubble with the stratosphere. The lowest contour value and the contour interval in (a) are 0.3 and $0.6 \mathrm{~K}$, respectively. Shaded area in both panels is the initial position of the "stratosphere" with strong stability.

and pseudo-incompressible dynamical cores in the lower $45 \mathrm{~km}$ of the vertical domain. At first, the buoyant bubble rises freely through its neutrally stratified environment. The bubble reaches the "stratosphere" at all simulations (Figs. 11a,e,i,m). A negative potential temperature perturbation forms at the lower edge of the stable stratosphere due to the upward motion associated with the rising bubble. While the bubble spreads laterally and dissipates near the "tropopause," the waves generated by the collision of the bubble with the tropopause propagate deep into the stable stratosphere after 60 simulated minutes (Figs. 11d,h,p). The simulated wavelength, propagation speed, and amplitude of the gravity waves in the stratosphere are similar in the unified, fully compressible, and pseudo-incompressible simulations. In contrast, the gravity waves in the anelastic simulation are weak and hardly propagate into the stratosphere during this period (Figs. 11k,l). These results support the conclusions of normal mode analyses presented by Arakawa and Konor (2009). The unified system yields as accurate simulations of gravity wave propagation in a stable environment as the fully compressible system while the anelastic system simulates only weak wave activity. The pseudo-incompressible dynamical core also performs well in this test. We are currently conducting a more detailed comparison study of the simulations of motion for wide range of scales and depths, including large-scale $\beta$-plane simulations, with various systems.

\section{2) Cold Bubble simulations}

For comparison purposes, the two-dimensional cold bubble test case, which was originally used by Straka et al. (1993), is simulated with the unified and fully compressible dynamical cores. The same simulation setup is used with both models, that is, the same initial condition prescribed by Straka et al. (1993) and second-order diffusion with a coefficient of $75 \mathrm{~m}^{2} \mathrm{~s}^{-1}$. The potential temperature and horizontal and vertical velocities are diffused in the unified and fully compressible models.

The results presented here were obtained by using 50-m grid distance in the horizontal and vertical directions. 

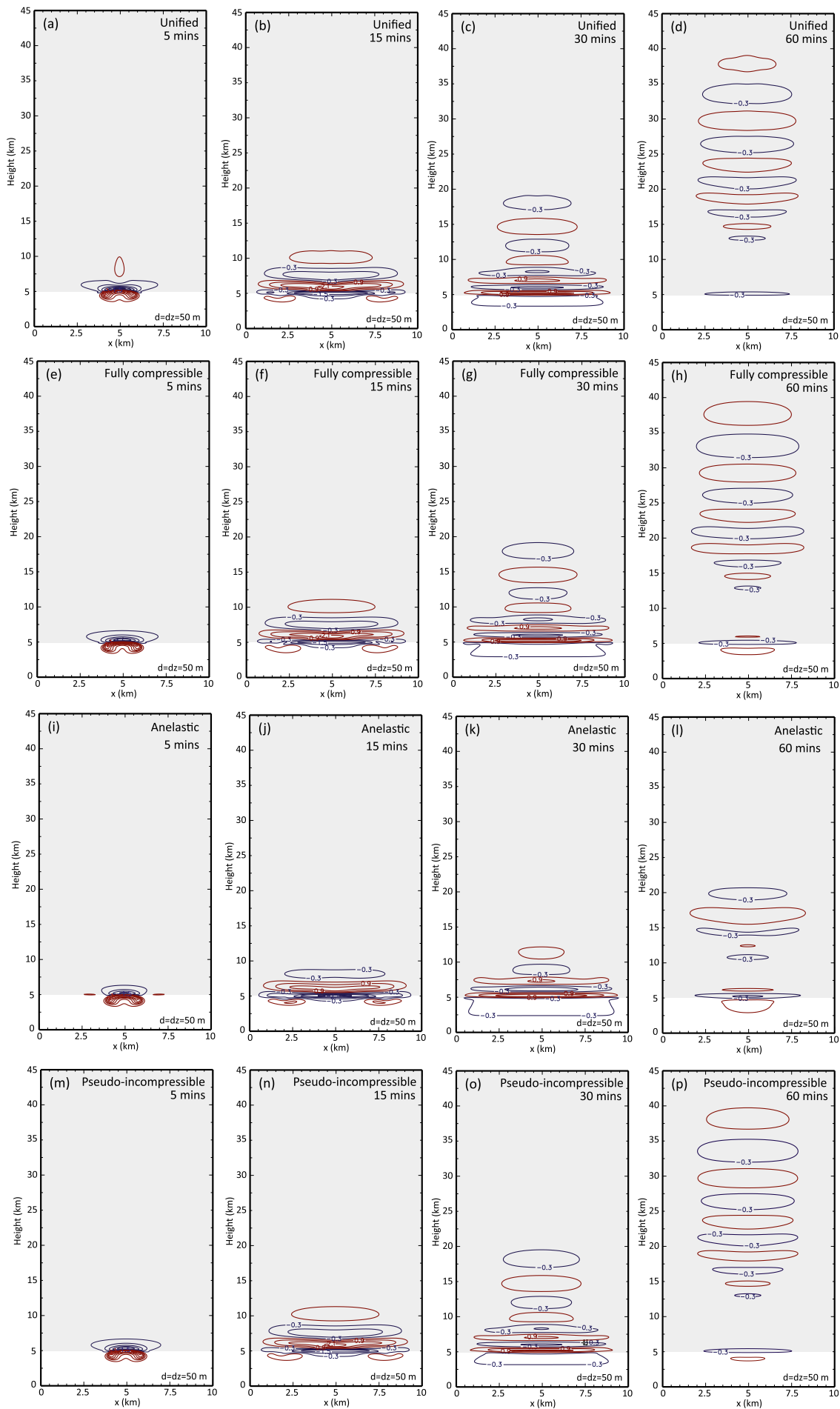

FIG. 11. The potential temperature perturbation (K) at (left to right) 5, 15, 30, and $60 \mathrm{~min}$ from (a)-(d) the unified, (e)-(h) the fully compressible, (i)-(l) anelastic, and (m)-(p) pseudoincompressible dynamical cores. One of the contour values and the contour interval are 0.3 and $0.6 \mathrm{~K}$, respectively. Red and blue lines are positive and negative values, respectively. Shaded area is the initial position of the "stratosphere" with strong stability. 
(a)

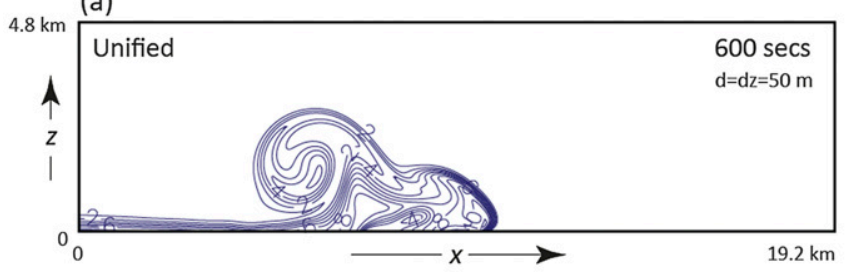

(c)

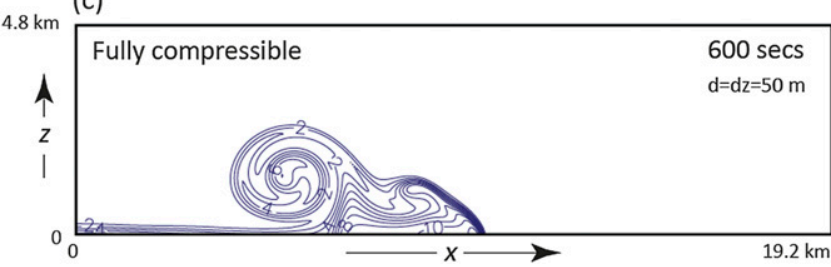

(b)

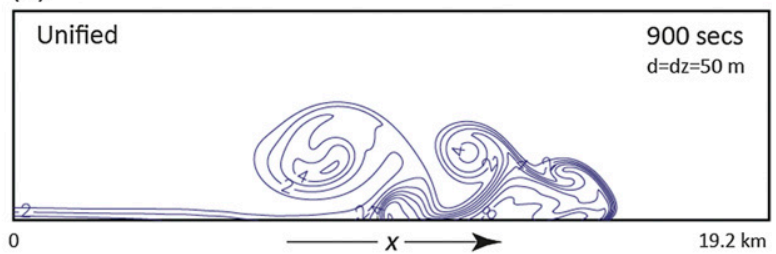

(d)

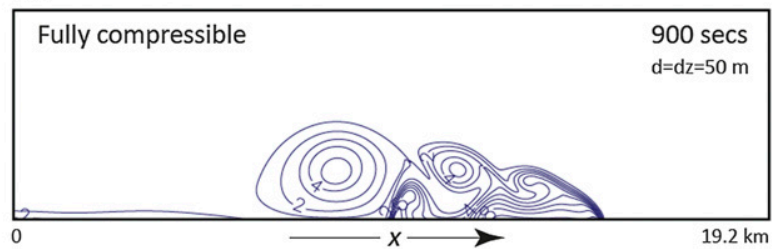

FIG. 12. The potential temperature perturbation (K) at (left) 600 and (right) 900 s of integration, from (a),(b) the unified and (c),(d) the fully compressible dynamical cores. Note that contour labels are multiplied by $(-1)$. The lowest contour value and the contour interval are both $1 \mathrm{~K}$.

The time step was $0.5 \mathrm{~s}$ in the unified dynamical core and 100 iterations in the elliptic solver are needed for this simulation. Figure 12 shows the simulated potential temperature perturbation at 600 and $900 \mathrm{~s}$ for the righthand side of the domain as obtained with the unified (Figs. 12a,b), and the fully compressible (Figs. 12c,d) dynamical cores. As expected, the consecutive development of intense vertical vortices resulting from the Kelvin-Helmholtz shear instability is evident at the upper edge of the cold outflow at 600 and $900 \mathrm{~s}$ into the integration. The results obtained with the two models agree overall although the outflow front moves slightly faster in the unified dynamical core than that in the fully compressible dynamical core. Also, in the tail of the cold outflow, the surface air is slightly colder in the unified simulation than in the fully compressible simulation.

\section{Summary, conclusions, and future directions}

We have presented the design of a dry dynamical core based on the nonhydrostatic unified system of equations. The unified system filters vertically propagating acoustic waves. The dynamical core predicts the potential temperature and horizontal momentum without any approximation. The quasi-hydrostatic Exner pressure and density are determined from the predicted potential temperature. In the continuity equation, the density is replaced by the "quasi-hydrostatic density"; this is the only approximation made. Since the quasi-hydrostatic density is determined from the predicted potential temperature, the continuity equation is not a prognostic equation; instead, it is used to diagnostically determine the vertical momentum. The time derivative of the quasi-hydrostatic density in the continuity equation is determined through a finite-difference scheme that uses the values of the quasi-hydrostatic density from the previous two time steps.

High-order upstream finite-difference schemes are used to discretize scalar advection. The discrete forms of horizontal mass and mass-weighted potential temperature fluxes are based on the third-order scheme of Takacs (1985). The vertical mass-weighted potential temperature fluxes are determined from another thirdorder finite-difference scheme. The horizontal momentum equation is discretized following Takano and Wurtele's (1982) fourth-order with a modification that guarantees dissipation of the (potential) enstrophy. The vertical advection of momentum is based on the second-order centered scheme. A fifth-order version of the scheme is also tested in the cold bubble test, but no significant difference is found in the solutions. Both the horizontal and vertical mass-weighed fluxes of vertical velocity are based on fifth-order upstream finitedifference schemes. For the time discretization of the thermodynamics and momentum equations, a secondorder Adams-Bashforth scheme is used. The time discretization of the pressure gradient force terms follow Mesinger and Arakawa's (1976) economical implicit time integration scheme.

The elliptic solver that used to determine the nonhydrostatic Exner pressure is the most computationally demanding part of the dynamical core. The solver used here is a block-Jacobi type, in which a simple iterative solver in the horizontal is combined with an implicit solver in the vertical. Certainly, there are more computationally efficient solvers available, but our solver performs well in these applications. Although there 
have been a lot of improvements in the design of elliptic solvers used in atmosphere models (e.g., Fulton et al. 1986; Smolarkiewicz and Margolin 1994; Smolarkiewicz et al. 1997; Skamarock et al. 1997; Heikes and Randall 1995; Heikes et al. 2013; and others), more work is still needed to test and compare the performance of different methods under various conditions with different grids, global and limited domains, and lateral boundaries and surface topography. One of the future developments of this dynamical core should be the improvement of the elliptic solver.

The integration procedure presented in section 3 has been shown to work successfully for a wide range of atmospheric scales. Idealized extratropical cyclogenesis simulations with different horizontal resolutions, including the case suggested by Ullrich and Jablonowski (2012), show that the dynamical core is able to successfully capture the details of the extratropical cyclogenesis and the associated frontogenesis process at the surface and in the mid- and upper troposphere. Cloud-scale warm and cold bubble tests have also been successfully carried out. The warm and cold bubble solutions obtained with the unified dynamical core compare well to those obtained with a fully compressible dynamical core.

This paper does not present a comprehensive and methodical comparison of solutions obtained with the unified dynamical core to those obtained with the fully compressible, anelastic, pseudo-incompressible, and quasi-hydrostatic dynamical cores. Such a comparison study must be carefully conducted and must cover a wide variety of cases including those designed to test global dynamical cores.

We acknowledge that there could be different integration algorithms for the solution of the unified system of equations. In the search for a better algorithm, special attention should be given to allowing longer time steps.

Although the unified dynamical core presented in this paper is based on the height coordinate, the unified system can use a generalized vertical coordinate such as the ones developed by Konor and Arakawa (1997) and Toy and Randall (2009). A generalized coordinate can be designed to behave like sigma near the surface and like theta above, with a smooth transition in between. By using a terrain-following coordinate, topography can be included although the performance of the sigma coordinate with very steep topography needs to be evaluated.

Another approach to include topography is to replace the momentum-based dynamical core with the vector-vorticity dynamical core developed by Jung and Arakawa (2008). The use of the unified system with the vector-vorticity dynamics was already discussed by
Arakawa and Konor (2009). In the vector-vorticity unified dynamical core, topography can be implemented through "block mountain" method following Wu and Arakawa (2011), who showed successful simulations of flow over idealized steep topography. Other approaches, such as those of Adcroft et al. (1997) and Klemp (2011) will also be considered.

Acknowledgments. The author has greatly benefited from collaboration with Professors Akio Arakawa, David Randall, Ross Heikes, and Joon-Hee Jung throughout this research. The useful comments and suggestions on the manuscript by David Randall are highly appreciated. I also thank Prof. Rupert Klein and an anonymous reviewer for their constructive reviews. This research has been funded by the U.S. DOE under Cooperative Agreement DE-FC02-06ER64302 to Colorado State University, the DOE under DESC07050, the NSF Grant AGS-1062468, and the NSF Science and Technology Center for Multiscale Modeling of Atmospheric Processes, managed by Colorado State University under Cooperative Agreement ATM0425247.

\section{APPENDIX A}

\section{Discretization of Thermodynamic Equation}

The thermodynamic equation in Eq. (2.5) on the grid shown in Fig. 1 is discretized as

$$
\begin{aligned}
\theta_{i+1 / 2, j+1 / 2, k}^{(n+1)}= & \theta_{i+1 / 2, j+1 / 2, k}^{(n)}+\delta t\left(G_{\theta}\right)_{i+1 / 2, j+1 / 2, k}^{(n+1 / 2)} \\
& +\delta t\left(\frac{Q}{c_{p} \pi}\right)_{i+1 / 2, j+1 / 2, k}^{(n)}
\end{aligned}
$$

where the superscripts $(n)$ and $(n+1)$ denote the current time step, at which the values are known, and next time steps, for which the predictions are made, respectively. The time step $(n+1 / 2)$ denotes a time level between steps $n+1$ and $n$. In Eq. (A.1), $\delta t$ is the time step and $\left(G_{\theta}\right)_{i+1 / 2, j+1 / 2, k}^{(n+1 / 2)}$ is the advective tendency of potential temperature. Following the second-order Adams-Bashforth time integration scheme, the advective tendency is defined as

$$
\left(G_{\theta}\right)_{i+1 / 2, j+1 / 2, k}^{(n+1 / 2)}=a\left(G_{\theta}\right)_{i+1 / 2, j+1 / 2, k}^{(n)}+b\left(G_{\theta}\right)_{i+1 / 2, j+1 / 2, k}^{(n-1)},
$$

where $a=1.5$ and $b=-0.5$. In Eq. (3.2), $\left(G_{\theta}\right)_{i+1 / 2, j+1 / 2, k}^{(n)}$ is the combination of horizontal and vertical advective tendencies written by 


$$
\begin{aligned}
\left(G_{\theta}\right)_{i+1 / 2, j+1 / 2, k}^{(n)}= & -\frac{\left[\nabla_{H} \cdot\left(\theta \rho_{\mathrm{qs}} \mathbf{v}\right)_{k}\right]_{i+1 / 2, j+1 / 2}^{(n)}-\theta_{i+1 / 2, j+1 / 2, k}^{(n)}\left[\mathbf{\nabla}_{H} \cdot\left(\rho_{\mathrm{qs}} \mathbf{v}\right)_{k}\right]_{i+1 / 2, j+1 / 2}^{(n)}}{\left(\rho_{\mathrm{qs}}\right)_{i+1 / 2, j+1 / 2, k}^{(n)}} \\
& -\frac{\left(\theta \rho_{\mathrm{qs}} w\right)_{i+1 / 2, j+1 / 2, k+1 / 2}^{(n)}-\left(\theta \rho_{\mathrm{qs}} w\right)_{i+1 / 2, j+1 / 2, k-1 / 2}^{(n)}}{\left(\rho_{\mathrm{qs}}\right)_{i+1 / 2, j+1 / 2, k}^{(n)}(\delta z)_{k}} \\
& +\theta_{i+1 / 2, j+1 / 2, k}^{(n)} \frac{\left(\rho_{\mathrm{qs}} w\right)_{i+1 / 2, j+1 / 2, k+1 / 2}^{(n)}-\left(\rho_{\mathrm{qs}} w\right)_{i+1 / 2, j+1 / 2, k-1 / 2}^{(n)}}{\left(\rho_{\mathrm{qs}}\right)_{i+1 / 2, j+1 / 2, k}^{(n)}(\delta z)_{k}} .
\end{aligned}
$$

This form is an advective form obtained from the flux convergence of potential temperature and mass. When time is continuous, Eq. (A.3) conserves massweighted potential temperature while maintaining the monotonicity of potential temperature. The
$\left(G_{\theta}\right)_{i+1 / 2, j+1 / 2, k}^{(n-1)}$ is saved from $\left(G_{\theta}\right)_{i+1 / 2, j+1 / 2, k}^{(n)}$ from one time step to the next. In Eq. (A.3), the horizontal mass-weighted potential temperature and the horizontal mass convergence terms are horizontally discretized by

$$
\left[\nabla_{H} \cdot\left(\theta \rho_{\mathrm{qs}} \mathbf{v}\right)_{k}\right]_{i+1 / 2, j+1 / 2}^{(n)} \equiv \frac{\left(\theta \rho_{\mathrm{qs}} u\right)_{i+1, j+1 / 2, k}^{(n)}-\left(\theta \rho_{\mathrm{qs}} u\right)_{i, j+1 / 2, k}^{(n)}+\left(\theta \rho_{\mathrm{qs}} v\right)_{i+1 / 2, j+1, k}^{(n)}-\left(\theta \rho_{\mathrm{qs}} v\right)_{i+1 / 2, j, k}^{(n)}}{d}
$$

and

$$
\left[\nabla_{H} \cdot\left(\rho_{\mathrm{qs}} \mathbf{v}\right)_{k}\right]_{i+1 / 2, j+1 / 2}^{(n)} \equiv \frac{\left(\rho_{\mathrm{qs}} u\right)_{i+1, j+1 / 2, k}^{(n)}-\left(\rho_{\mathrm{qs}} u\right)_{i, j+1 / 2, k}^{(n)}+\left(\rho_{\mathrm{qs}} v\right)_{i+1 / 2, j+1, k}^{(n)}-\left(\rho_{\mathrm{qs}} v\right)_{i+1 / 2, j, k}^{(n)}}{d},
$$

respectively. See the supplementary material for the definition of the third-order vertical and horizontal fluxes used in Eqs. (A.3), (A.4a), and (A.4b).

\section{APPENDIX B}

\section{Derivation of the Expression for the Quasi-Hydrostatic Exner Pressure at the Model Layers}

To find the expression that interpolates the quasihydrostatic Exner pressure from interfaces to the model layers, the vertically and temporally discrete equation in Eq. (2.9a) with Eq. (2.13) is written as

$$
\begin{aligned}
\left(p_{\mathrm{qs}}\right)_{i+1 / 2, j+1 / 2, k-1 / 2}^{(n+1)}= & \left(p_{\mathrm{qs}}\right)_{i+1 / 2, j+1 / 2, k+1 / 2}^{(n+1)} \\
& +g \frac{p_{00}\left[\pi_{\mathrm{qs}}^{(1-\kappa) / \kappa}\right]_{i+1 / 2, j+1 / 2, k}^{(n+1)}}{R \theta_{i+1 / 2, j+1 / 2, k}^{(n+1)}}(\delta z)_{k} .
\end{aligned}
$$

Then Eq. (2.9b) is discretized as

$$
\begin{aligned}
\left(\pi_{\mathrm{qs}}\right)_{i+1 / 2, j+1 / 2, k-1 / 2}^{(n+1)}= & \left(\pi_{\mathrm{qs}}\right)_{i+1 / 2, j+1 / 2, k+1 / 2}^{(n+1)} \\
& +\frac{g}{c_{p}} \frac{(\delta z)_{k}}{\theta_{i+1 / 2, j+1 / 2, k}^{(n+1)}} .
\end{aligned}
$$

By eliminating $(\delta z)_{k} / \theta_{i+1 / 2, j+1 / 2, k}^{(n+1)}$ between Eqs. (B.1a) and (B.2b) and using $\kappa \equiv R / c_{p}$, we find a formula that is used for the interpolation of the quasi-hydrostatic Exner pressure from interfaces to the model layers as

$$
\left(\pi_{\mathrm{qs}}\right)_{i+1 / 2, j+1 / 2, k}^{(n+1)}=\left[\frac{\kappa}{p_{00}} \frac{\left(p_{\mathrm{qs}}\right)_{k-1 / 2}^{(n+1)}-\left(p_{\mathrm{qs}}\right)_{k+1 / 2}^{(n+1)}}{\left(\pi_{\mathrm{qs}}\right)_{k-1 / 2}^{(n+1)}-\left(\pi_{\mathrm{qs}}\right)_{k+1 / 2}^{(n+1)}}\right]_{i+1 / 2, j+1 / 2}^{[\kappa /(1-\kappa)]} .
$$

\section{REFERENCES}

Abiodun, B. J., J. M. Prusa, and W. J. Gutowski, 2008: Implementation of a non-hydrostatic, adaptive-grid dynamics core in CAM3. Part I: Comparison of dynamics cores in aquaplanet simulations. Climate Dyn., 31, 795-810.

Adcroft, A., C. Hill, and J. Marshall, 1997: The representation of topography by shaved cells in a height coordinate ocean model. Mon. Wea. Rev., 125, 2293-2315.

Arakawa, A., 1988: Finite-difference methods in climate modeling. Physically Based Modelling and Simulation of Climate and Climate Change, Part I, M. E. Schlesinger, Ed., Kluwer Academic Publisher, 79-168.

_ , and V. R. Lamb, 1977: Computational design of the basic dynamical processes of the UCLA general circulation model. Methods in Computational Physics, J. Chang, Ed., Vol. 17, Academic Press, 173-265. 
and C. S. Konor, 1996: Vertical differencing of the primitive equations based on a Charney-Phillips type grid in a $\sigma-p$ hybrid vertical coordinates. Mon. Wea. Rev., 124, 511-528.

—- and — 2009: Unification of the anelastic and quasihydrostatic systems of equations. Mon. Wea. Rev., 137, 710-726.

Bannon, P. R., 1996: On the anelastic approximation for a compressible atmosphere. J. Atmos. Sci., 53, 3618-3628.

Bonaventura, L., 2004: The ICON project: Development of a unified model using triangular geodesic grids. Proc. ECMWF Seminar on Recent Developments in Numerical Methods for Atmosphere and Ocean Modelling, Shinfield Park, Reading, United Kingdom, ECMWF, 75-86.

Côté, J., S. Gravel, A. Méthot, A. Patoine, M. Roch, and A. Staniforth, 1998: The operational CMC-MRB Global Environmental Multiscale (GEM) model. Part I: Design considerations and formulation. Mon. Wea. Rev., 126, 1373-1395.

Cullen, M. J. P., 1990: A test of a semi-implicit integration technique for a fully compressible non-hydrostatic model. Quart. J. Roy. Meteor. Soc., 116, 1253-1258, doi: 10.1002/qj.49711649513.

Davies, T., A. Staniforth, N. Wood, and J. Thuburn, 2003: Validity of anelastic and other equation sets as inferred from normalmode analysis. Quart. J. Roy. Meteor. Soc., 129, 2761-2775, doi:10.1256/qj.02.1951.

—, M. J. P. Cullen, A. J. Malcolm, M. H. Mawson, A. Staniforth, A. A. White, and N. Wood, 2005: A new dynamical core for the Met Office's global and regional modelling of the atmosphere. Quart. J. Roy. Meteor. Soc., 131, 1759-1782, doi: 10.1256/qj.04.101.

Dukowicz, J., 2013: Evaluation of various approximations in atmosphere and ocean modeling based on an exact treatment of gravity wave dispersion. Mon. Wea. Rev., in press.

Durran, D. R., 1989: Improving the anelastic approximation. J. Atmos. Sci., 46, 1453-1461.

_ 2008: A physically motivated approach for filtering acoustic waves from the equations governing compressible stratified flow. J. Fluid Mech., 601, 365-379.

Fulton, S. R., P. E. Ciesielski, and W. Schubert, 1986: Multigrid methods for elliptic problems: A review. Mon. Wea. Rev., 114, 943-959.

Gallus, W. A., and M. Rancic, 1996: A non-hydrostatic version of the NMC's regional Eta model. Quart. J. Roy. Meteor. Soc., 122, 495-513, doi:10.1002/qj.49712253010.

Grabowski, W. W., and T. L. Clark, 1991: Cloud environment interface instability: Rising thermal calculations in two spatial dimensions. J. Atmos. Sci., 48, 527-546.

Heikes, R. P., and D. A. Randall, 1995: Numerical integration of the shallow water equations on a twisted icosahedral grid. Part I: Basic design and results of tests. Mon. Wea. Rev., 123, 1862-1880.

$\longrightarrow, \ldots$, and C. S. Konor, 2013: Optimized icosahedral grids: Performance of finite-difference operators and multigrid solver. Mon. Wea. Rev., in press.

Janjic, Z. I., 2003: A nonhydrostatic model based on a new approach. Meteor. Atmos. Phys., 82, 271-285.

— J. P. Gerrity Jr., and S. Nickovic, 2001: An alternative approach to nonhydrostatic modeling. Mon. Wea. Rev., 129, 1164-1178.

Jung, J.-H., and A. Arakawa, 2008: A three-dimensional anelastic model based on the vorticity equation. Mon. Wea. Rev., 136, 276-294.

Klein, R., U. Achatz, D. Bresch, O. M. Knio, and P. K. Smolarkiewicz, 2010: Regime of validity of soundproof atmospheric flow models. J. Atmos. Sci., 67, 3226-3237.

Klemp, J. B., 2011: A terrain-following coordinate with smoothed coordinate surfaces. Mon. Wea. Rev., 139, 2163-2169.
_ , and R. Wilhelmson, 1978: The simulation of three-dimensional convective storm dynamics. J. Atmos. Sci., 35, 1070-1096.

Konor, C. S., and A. Arakawa, 1997: Design of an atmospheric model based on a generalized vertical coordinate. Mon. Wea. Rev., 125, 1649-1673.

Kurowski, M. J., W. W. Grabowski, and P. K. Smolarkiewicz, 2013: Toward multiscale simulation of moist flows with soundproof equations. J. Atmos. Sci., in press.

Kwizak, M., and A. J. Robert, 1971: A semi-implicit scheme for grid point atmospheric models of the primitive equations. Mon. Wea. Rev., 99, 32-36.

Laprise, R., 1992: The Euler equations of motion with hydrostatic pressure as an independent variable. Mon. Wea. Rev., 120, 197-207.

Lipps, F. B., and R. S. Hemler, 1982: A scale analysis of deep moist convection and some related numerical calculations. J. Atmos. Sci., 39, 2192-2210.

Lorenz, E. N., 1960: Energy and numerical weather prediction. Tellus, 12, 364-373.

Marshall, J., A. Adcroft, C. Hill, L. Perelman, and C. Heisey, 1997: A finite-volume, incompressible Navier-Stokes model for studies of the ocean on parallel computers. J. Geophys. Res., 102 (C3), 5753-5766.

Mendez-Nunez, L. R., and J. J. Carroll, 1994: Application of the MacCormack scheme to atmospheric nonhydrostatic models. Mon. Wea. Rev., 122, 984-1000.

Mesinger, F., and A. Arakawa, 1976: Numerical methods used in atmospheric models. GARP Publication Series 17, Part I $64 \mathrm{pp}$.

Miller, M. J., 1974: On the use of pressure as vertical co-ordinate in modeling convection. Quart. J. Roy. Meteor. Soc., 100, 155162, doi:10.1002/qj.49710042403.

Newton, C. W., and A. Trevisan, 1984: Clinogenesis and frontogenesis in jet-stream waves. Part II: Channel model numerical experiments. J. Atmos. Sci., 41, 2735-2755.

Ogura, Y., and N. A. Phillips, 1962: Scale analysis of deep and shallow convection in the atmosphere. J. Atmos. Sci., 19, 173-179.

Robert, A., 1982: A semi-Lagrangian and semi-implicit scheme for primitive equations. J. Meteor. Soc. Japan, 60, 319-324.

_ 1993: Bubble convection experiments with a semi-implicit formulation of the Euler equations. J. Atmos. Sci., 50, 18651873.

Satoh, M., T. Matsuno, H. Tomita, H. Miura, T. Nasuno, and S. Iga, 2008: Nonhydrostatic Icosahedral Atmospheric Model (NICAM) for global cloud resolving simulations. J. Comput. Phys., 227, 3486-3514, doi:10.1016/j.jcp.2007.02.006.

Shapiro, M. A., 1975: Simulation of upper-level frontogenesis with a 20-level isentropic coordinate primitive equation model. Mon Wea. Rev., 103, 591-604.

Skamarock, W. C., and J. B. Klemp, 2008: A time-split nonhydrostatic atmospheric model for weather research and forecasting applications. J. Comput. Phys., 227, 3465-3485.

_ , P. K. Smolorkiewicz, and J. B. Klemp, 1997: Preconditioned conjugate-residual solvers for Helmholtz equations in nonhydrostatic models. Mon. Wea. Rev., 125, 587-599.

—_, J. B. Klemp, J. Dudhia, D. Gill, D. Barker, W. Wang, and J. G. Powers, 2005: A description of the Advanced Research WRF version 2. NCAR Tech. Note NCAR/TN-468+STR, $88 \mathrm{pp}$.

,, M. G. Duda, L. Fowler, S.-H. Park, and T. D. Ringler, 2012: A multiscale nonhydrostatic atmospheric model using centroidal Voronoi Tessellations and C-grid staggering. Mon. Wea. Rev., 140, 3090-3105. 
Smolarkiewicz, P. K., 2010: Modeling atmospheric circulations with soundproof equations. Proc. ECMWF Workshop on Nonhydrostatic Modeling, Shinfield Park, Reading, United Kingdom, ECMWF, 15 pp.

—_, and J. A. Pudykiewicz, 1992: A class of semi-Lagrangian approximations for fluids. J. Atmos. Sci., 49, $2082-2096$.

—, and L. G. Margolin, 1994: Variational solver for elliptic problems in atmospheric flows. Appl. Math. Comput. Sci., 4, 527-551.

—, V. Grubisic, and L. G. Margolin, 1997: On forward-in-time differencing for fluids: Stopping criteria for iterative solutions of anelastic pressure equations. Mon. Wea. Rev., 125, 647-654.

— L. L. Gargolin, and A. A. Wyszogrodzki, 2001: A class of nonhydrostatic global models. J. Atmos. Sci., 58, 349-364.

Staniforth, A., A. White, J. Thuburn, M. Zerroukat, E. Cordero, and T. Davies, 2003: Joy of U.M. 5.5-model formulation. Unified Model Documentation Paper 15, Met Office, 530 pp.

Steppeler, J., R. Hess, U. Schättler, and L. Bonaventura, 2003: Review of numerical methods for nonhydrostatic weather prediction models. Meteor. Atmos. Phys., 82, 287-301.

Straka, J. M., R. B. Wilhelmson, L. J. Wicker, J. R. Anderson, and K. K. Droegemeier, 1993: Numerical solutions of a non-linear density current: A benchmark solution and comparisons. Int. J. Numer. Methods Fluids, 17, 1-22.
Takacs, L. L., 1985: A two-step scheme for the advection equation with minimized dissipation and dispersion errors. Mon. Wea. Rev., 113, 1050-1065.

Takano, K., and M. G. Wurtele, 1982: A fourth order energy and potential enstrophy conserving difference scheme. Air Force Geophysics Laboratory Rep. AFGL-TR-82-0205 (NTIS ADA126626), $87 \mathrm{pp}$.

Tapp, M., and P. W. White, 1976: A non-hydrostatic mesoscale model. Quart. J. Roy. Meteor. Soc., 102, 277-296, doi:10.1002/ qj. 49710243202.

Toy, M. D., and D. A. Randall, 2009: Design of a non-hydrostatic atmospheric model based on a generalized vertical coordinate. Mon. Wea. Rev., 137, 2305-2330.

Ullrich, P., and C. Jablonowski, 2012: Operator-split RungeKutta-Rosenbrock methods for nonhydrostatic atmospheric models. Mon. Wea. Rev., 140, 1257-1284.

Wu, C.-M., and A. Arakawa, 2011: Inclusion of surface topography into the vector vorticity equation model (VVM). J. Adv. Model. Earth Syst., 3, M06002, doi:10.1029/ 2011MS000061.

Yeh, K.-S., J. Côté, S. Gravel, A. Méthot, A. Patoine, M. Roch, and A. Staniforth, 2002: The CMC-MRB Global Environmental Multiscale (GEM) model. Part III: Nonhydrostatic formulation. Mon. Wea. Rev., 130, 339-356. 